ESAIM: PROCEEDINGS AND SURVEYS, June 2017, Vol. 56, p. 1-21

S. Crépey, M. Jeanblanc and A. Nikeghbali Editors

\title{
FROM THE DECOMPOSITIONS OF A STOPPING TIME TO RISK PREMIUM DECOMPOSITIONS*
}

\author{
Delia Coculescu ${ }^{1}$
}

\begin{abstract}
The occurrence of some events can impact asset prices and produce losses. The amplitude of these losses are partly determined by the degree of predictability of those events by the market investors, as risk premiums build up in an asset price as a compensation of the anticipated losses. The aim of this paper is to propose a general framework where these phenomena can be properly defined and quantified.

Our focus are the default events and the defaultable assets, but the framework could apply to any event whose occurrence impacts some asset prices.

We provide the general construction of a default time under the so called $(\mathbf{H})$ hypothesis, which reveals a useful way in which default models can be built, using both market factors and idiosyncratic factors. All the relevant characteristics of a default time (i.e. the Azéma supermartingale and its Doob-Meyer decomposition) are explicitly computed given the information about these factors.

We then define the default event risk premiums and the default adjusted probability measure. These concepts are useful for pricing defaultable claims in a framework that includes possible economic shocks, such as jumps of the recovery process or of some default-free assets at the default time. These formulas are not classic and we point out that the knowledge of the default compensator (or the intensity process when the default time is totally inaccessible) is not a sufficient quantity for finding explicit prices; the Azéma supermartingale and its Doob-Meyer decomposition are needed. The progressive enlargement of a filtration framework is the right tool for pricing defaultable claims in non standard frameworks where non defaultable assets or recovery processes may react at the default event.
\end{abstract}

\section{INTRODUCTION}

Negative financial events such as defaults can sometimes be predicted by investors or, on the opposite, they can occur in an abrupt way and produce losses. In this paper, the properties of a stopping time which models a default event are analyzed in relation with the losses that it produces to debt-holders when it occurs, using standard properties of the jump times of martingales.

In the "general theory of processes", one classifies stopping times as predictable, accessible and totally inaccessible stopping times (see Definition 2.1 below). Traditionally, in the default risk literature structural models have produced default times that are predictable stopping times (for instance the first hitting time of a fixed level by a diffusion), whereas in the reduced form approach defaults are modeled as totally inaccessible stopping times (first jump of a Cox process). The difference between the two classes of stopping times can be

* I would like to thank Monique Jeanblanc and an anonymous referee for their comments and suggestions which helped to improve the present text.

${ }^{1}$ Department of Banking and Finance

University of Zürich, Plattenstrasse 32

Zürich 8032, Switzerland.

(C) EDP Sciences, SMAI 2017 
eliminated by a change of the underlying filtration: a predictable stopping time can become totally inaccessible in a smaller filtration, as illustrated by several so-called incomplete information models including [16], [29], [23]. See also the paper [22] where this information-based connection between the structural and reduced-form approaches is explained. Using no arbitrage arguments, we show (Section 2) that defaults that are thought to produce losses for a financial asset do not have a predictable part and it is hence natural to model them as totally inaccessible stopping times in the market filtration.

It has become standard to construct reduced-form default models in two steps (as originally proposed in [17], [24]): one begins with a filtration where the default time is not observable, and then obtain the market filtration after progressively enlarging the original filtration so that the default time becomes a stopping time. In Section 3 we present this construction and some fundamental results that we shall subsequently use in the paper. Our leading assumption in this paper will be that in the enlarged filtration the martingales from the original filtration remain martingales, a property known in the literature as the (H) hypothesis.

In Section 4 we study some general properties of a stopping time $\tau$ (i.e., the default time) under this two-step construction. In particular, we emphasize that there exist decomposing sequences involving some stopping times $\left(T^{i}\right), i \geq 1$ of the initial filtration $\mathbb{F}$ and a random time avoiding the $\mathbb{F}$ stopping times $T^{0}$ (Proposition 4.2 ). We use the properties of these times (i.e., their compensators) in order to characterize the Azéma supermartingale of the default time and give its Doob-Meyer decomposition (Proposition 4.10). This represents a generalization of the classical models, where the Azéma supermartingale is supposed to be continuous. The particular case when $\tau$ is totally inaccessible is also analyzed and we give a useful economic interpretation to this decomposition.

In Section 5, we apply our results (in particular the decompositions studied in Section 4) in order to obtain pricing formulas for defaultable claims. The usual reduced-form framework is extended in order to include possible economic shocks, and in particular jumps of the recovery process at the default time. Indeed, there has been increasing support in the empirical literature that both the probability of default and the loss given default are correlated and driven by macroeconomic variables (see [2], [6]). A perfect illustration of this phenomenon is the rapid decline in property prices the 2008 US mortgage credit crisis, where defaults coincided with a wave of asset liquidation. Our aim is to extend the usual reduced-form setup in order to include possible jumps in the recoveries at the default time and to give an expression for the risk premiums attached to such jumps.

Building on the method developed in [10], we develop pricing formulas which are not classic (precisely because of the possible jumps of some default-free assets at the default time): the knowledge of the default compensator or the intensity process is not anymore a sufficient quantity for finding explicit prices, but we need indeed the Azéma supermartingale and its Doob-Meyer decomposition. We also propose a definition for the default event risk premium, which measures the compensation investors should require for the losses that occur at the default time; this turns out to be strictly positive when the default time is totally inaccessible, but null for a predictable default time. We provide the expression of the default event risk premium in a general setting (Theorem 5.8).

Addendum. The current paper is based on an older preprint (arXiv:0912.4312v2, 2010). Some subsequent research has appeared since. We mention below the most relevant ones.

The paper [28] proposes a model for sovereign bonds; the model is a beautiful illustration of how the decomposition we propose in Theorem 4.2 can be effectively used to construct economically meaningful default times. Also this study makes some steps outside the $(\mathbf{H})$ hypothesis, by proposing as well a construction under the so called density approach.

In [19], the authors introduce a framework for pricing zero recovery defaultable claims. They model directly the compensator of the default time, without relying on the Azéma supermartingale. The compensator proposed is a special form of our expression (4.7), where the economic shocks $\left(T^{i}\right), i \geq 1$ are predictable times and the idiosyncratic time, which is totally inaccessible stopping time $T^{0}$, admits an intensity. As a consequence, the default adjusted probability measure that we define in Definition 5.5 coincides when applied to the model in [19] with the risk neutral measure.

On the mathematical side, without having directly finance in view, Aksamit and al. [1] propose a general decomposition of a random time, that they call $(\star)$ decomposition, which is closely linked to the decompostions we propose in Proposition 4.2. In the $(\star)$ decomposition, a random time can be uniquely written as infimum 
of two other random times, one of them being the time we have denoted here $T^{0}$, and the other one can be constructed from any sequence we have denoted $\left(T^{i}\right), i \geq 1$.

\section{LOSSES AND THEIR PREDICTABILITY}

Let $\left(\Omega, \mathcal{F}, \mathbb{H}=\left(\mathcal{H}_{t}\right)_{t \geq 0}, \mathbf{P}\right)$ be a filtered probability space. The filtration $\mathbb{H}=\left(\mathcal{H}_{t}\right)_{t \geq 0}$ represents an information flow available to all market investors without cost, that is public information. $\tau$ is an $\mathbb{R}_{+}$valued random variable such that $\tau>0$ a.s. which represents the default time of a company, i.e., the time when the company is not able to meet some of its financial obligations. Since corporate defaults can be observed when they occur, $\tau$ is supposed to be an $\mathbb{H}$ stopping time.

We want to deduce some properties of the particular stopping time $\tau$, given the impact of the default on the prices of the traded assets or portfolios of assets which are available in a financial market. An important question is to know under which economic conditions $\tau$ should be modeled as a totally inaccessible stopping time. Let us recall below a classification of stopping times:

Definition 2.1. [Classification of stopping times] A stopping time $\tau$ is said to be:

(i) predictable if there exists a sequence of stopping times $\left(\tau_{n}\right)_{n \geq 1}$ such that $\tau_{n}$ is increasing, $\tau_{n}<\tau$ on $\{\tau>0\}$ for all $n$, and $\lim _{n \rightarrow \infty} \tau_{n}=\tau$ a.s..

(ii) accessible if there exists a sequence of predictable stopping times $\left(\tau_{n}\right)_{n \geq 1}$, such that:

$$
\mathbb{P}\left(\cup_{n}\left\{\omega: \tau(\omega)=\tau_{n}(\omega)<\infty\right\}\right)=1 .
$$

(iii) totally inaccessible if, for every predictable stopping time $T$,

$$
\mathbb{P}(\{\omega: \tau(\omega)=T(\omega)<\infty\})=0 .
$$

In the line of the now classical asset modeling and pricing, we consider a collection of $\mathbb{H}$-adapted processes $\left(S_{t}^{i}\right)_{t \geq 0}, i \in\{0,1, \ldots, n\}$ with right continuous and left hand limits sample paths, which represents the evolution of the prices of the assets which are traded in a financial market. Without any loss of generality, we can suppose in this section that the money market account $\left(S_{t}^{0}\right)$ is constant or, equivalently, that the interest rates are null. We suppose that prices are in equilibrium when regarded as stochastic processes in this filtration, that is, there exists a probability measure $\mathbf{Q} \sim \mathbf{P}$ such that $\left(S_{t}^{i}\right)$ is a locally bounded local martingale for any $i \in\{1, \ldots, n\}$.

We assume that there is at least one defaultable asset which is traded on the market (for instance a bond), whith price process $\left(S_{t}^{d}\right)$ for some $d \in\{1, \ldots, n\}$. Furthermore we make the following crucial assumption:

(L) Suppose that $\left(S_{t}^{d}\right)$ is the price of a defaultable claim. Assume that $\Delta S_{\tau}^{d}<0$ a.s., i.e., there is a loss in case of default with probability one.

Since the default of a debtor is perceived as a bad news for the creditors, it is reasonable to assume that bond prices or, more generally, prices of defaultable claims, will certainly decrease in the moments after the announcement of the default. Or, in a continuous time, arbitrage free market model (see [11]), prices decrease with certainty only by negative jumps. Hence, it is reasonable to assume that prices of defaultable claims display negative jumps at the default time. Symmetrically, short positions in defaultable claims would display positive jumps.

We show below that the characteristics of a default time of being predictable or not are in fact intimately related to the validity of the assumption $(\mathbf{L})$. Therefore, in practical applications, the debate about the properties of a default time can be oriented towards clarifications of the validity of the assumption (L), which can be tested using market data.

First let us introduce the restrictions of a stopping time as follows: if $\tau$ is a stopping time and $E \in \mathcal{F}$, then the restriction of $\tau$ to $E$ is

$$
\tau(E):=\tau \mathbf{1}_{E}+\infty \mathbf{1}_{E^{c}} .
$$

Notice $\tau(E)$ is a stopping time if and only if $E \in \mathcal{H}_{\tau}$.

We now recall the decomposition of a stopping time. 
Theorem 2.2 ( [12]). Let $\tau$ be an $\mathbb{H}$ - finite stopping time. There exists an essentially unique partition of $\Omega$ in two elements $A$ and $B$ of $\mathcal{H}_{\tau-}$ such that the time $\tau(A)$ is accessible and $\tau(B)$ is totally inaccessible. Hence:

$$
\tau=\tau(A) \wedge \tau(B)
$$

$\tau(A)$ is called the accessible part of $\tau$ and $\tau(B)$ is called the totally inaccessible part of $\tau$.

Remark. Let us point out that the decomposition (2.1) is stable under equivalent changes of the probability measure.

Proposition 2.3. Under $(\boldsymbol{L})$, the default time $\tau$ does not have a predictable part, that is, there does not exist $E \in \mathcal{H}_{\tau}$ such that $\tau(E)$ is a finite predictable stopping time. In particular, when it is finite, the accessible part of $\tau$ is not predictable.

Proof. It is equivalent to show the result under an equivalent local martingale measure for $S^{d}$, i.e., such that the process $\left(S_{t}^{d}\right)$ is a local martingale. Therefore we assume without loss of generality that $\mathbf{P}$ is a local martingale measure. Denote $\delta:=\Delta S_{\tau}^{d}$. By (L), $\delta<0$ a.s..

Suppose that there exists a set $E \in \mathcal{H}_{\tau}$ such that $\tau(E)$ is a predictable stopping time. Then we need to show that $\mathbf{P}(E)=0$ hence $\tau(E)=\infty$ a.s..

Let $\left(\theta^{k}\right)_{k \in \mathbb{N}}$ be a reducing sequence of stopping times for $\left(S_{t}^{d}\right)$, that is $\left(\theta^{k}\right)$ increases to $\infty$ and the stopped process $S_{t}^{d, k}:=S_{t \wedge \theta^{k}}^{d}$ is a uniformly integrable martingale. The predictable stopping theorem states that:

$$
\mathbf{E}\left[\Delta S_{\tau(E)}^{d, k} \mid \mathcal{H}_{\tau(E)-}\right]=0 \text { and implies that } \mathbf{E}\left[\Delta S_{\tau(E)}^{d, k}\right]=0
$$

Notice that $\Delta S_{\tau(E)}^{d, k}=\delta \mathbf{1}_{E} \mathbf{1}_{\left\{\tau \leq \theta^{k}\right\}}$ therefore we must have:

$$
\mathbf{E}\left[\delta \mathbf{1}_{E \cap\left\{\tau \leq \theta^{k}\right\}}\right]=0 .
$$

Since $\delta$ is strictly negative it follows that

$$
\mathbf{P}\left(E \cap\left\{\tau \leq \theta^{k}\right\}\right)=0 \text { for all } k \in \mathbb{N}
$$

Notice that $\left\{\tau \leq \theta^{k}\right\} \subset\left\{\tau \leq \theta^{k+1}\right\}$ hence taking the limit in (2.2) as $n \rightarrow \infty$ leads to $P(E)=0$.

The above proposition reveals that the structural models of default are not compatible with the assumption (L), since the default times in these models are modeled as hitting times of some observable (i.e., $\mathbb{H}$ adapted) diffusions, or jump diffusions, hence have a predictable part. A way to obtain the condition (L) from a structural model is to assume that the process triggering the default is not $\mathbb{H}$ adapted or in other words is not observable by common market investors (see for instance the incomplete information models in [16], [20], [7], [21], [8], [18]). A general discussion about the links between structural, reduced-form and imperfect information models is provided in [22].

It seems that totally inaccessible stopping times are suitable to be used when one is interested to model situations characterized in the assumption $(\mathbf{L})$. Let us point out that under $(\mathbf{L})$ the default time can nevertheless have an accessible part in some types of filtrations, since there exist in general accessible stopping times which are not predictable.

Corollary 2.4. Suppose that the filtration $\mathbb{H}$ is quasi left-continuous i.e., $\mathcal{H}_{S}=\mathcal{H}_{S-}$ for all predictable stopping times $S$. Under $(\boldsymbol{L})$, the default time $\tau$ is a totaly inacessible stopping time.

Proof. If the filtration $\mathbb{H}$ is quasi left-continuous, then all accessible stopping times are predictable (T51, page $62,[12])$.

Quasi left continuity of a filtration is not a very intuitive notion form an economical point of view. Let us point out that $\mathbb{H}$ is quasi left-continuous for instance when the filtration is generated by a Lévy process, 
this being the class of models the most commonly used in modeling security prices. Therefore, the above result suggest that different filtrations (i.e., which are not quasi left-continuous) should be used if one wants to capture some specific features of debt, such that possibilities to default at some fixed dates in time together with the assumption (L). In this case, accessible but not predictable stopping times should be used. We provide below an example of such a construction:

Example 2.5. Suppose that a bond is expected to pay coupons at the fixed dates $t^{i}, i=1, \ldots, n$ and the bond can default only at one of the coupon dates. We begin with a filtration $\mathbb{F}$ and a sequence $\left(b^{i}\right)_{i \geq 1}$ of Bernoulli random variables independent from each other and independent from the filtration $\mathbb{F}$. We denote $i^{*}:=\inf \left\{i \mid b^{i}=1\right\}$, the default time: $\tau=t^{i^{*}} \mathbf{1}_{\left\{i^{*} \leq n\right\}}+\infty \mathbf{1}_{\left\{i^{*}>n\right\}}$ and the market filtration: $\mathcal{H}_{t}:=\mathcal{F}_{t} \vee \sigma(\tau \wedge t)$ for $t \geq 0$. It is easy to check that the default time $\tau$ is an accessible but not predictable $\mathbb{H}$-stopping time, so that the assumption (L) can be implemented in this setting.

\section{ThE TWO-STEP CONSTRUCTION OF THE INFORMATION SET}

In this short section we fix the mathematical framework that will be used for the rest of the paper. Following [4], [17], [24], we shall construct a default model in two steps. The main idea is to separate those assets which do not default at time $\tau$, from those who are defaultable at $\tau$. Defaultable assets are those issued by the particular company we are analyzing, while the remaining traded assets (that, to simplify, we call default-free) are issued by other companies or by governments, they may as well be indexes, commodities or derivatives: they should all have different default times than $\tau$. Let $\left(S^{i}\right)_{i \in I}$, with $I \subset\{1, \ldots, n\}$ represent the price processes of the default-free assets and $\mathbb{F}$ their natural $\mathbf{P}$ augmented filtration. The default model is built using the information $\mathbb{F}$ about the default-free assets (instead of all the public information $\mathbb{H}$ ) and the default arrival as follows.

Let $\left(\Omega, \mathcal{F}, \mathbb{F}=\left(\mathcal{F}_{t}\right)_{t \geq 0}, \mathbf{P}\right)$ be a filtered probability space satisfying the usual assumptions. The default time $\tau$ is defined as a random time (i.e., a nonnegative $\mathcal{F}$-measurable random variable) which is not an $\mathbb{F}$-stopping time. We assume throughout that $\mathbf{P}(\tau=\infty)=0$.

Then, a second filtration $\mathbb{G}=\left(\mathcal{G}_{t}\right)_{t \geq 0}$ is obtained by progressively enlarging the filtration $\mathbb{F}$ with the random time $\tau: \mathbb{G}$ is the smallest filtration satisfying the usual assumptions, containing the original filtration $\mathbb{F}$, and for which $\tau$ is a stopping time, such as explained in [25], [27]:

$$
\mathcal{G}_{t}=\mathcal{K}_{t+}^{o} \quad \text { where } \quad \mathcal{K}_{t}^{o}=\mathcal{F}_{t} \vee \sigma(\tau \wedge t)
$$

The advantage of this construction is that it allows to rely on projections of some $\mathbb{G}$ adapted processes onto the filtration $\mathbb{F}$ and therefore displays some finer properties of the default time, which are very useful for pricing and hedging.

More precisely, the following $\mathbb{F}$ adapted processes shall play a crucial role in our discussion:

- the $\mathbb{F}$ supermartingale

$$
Z_{t}^{\tau}=\mathbf{P}\left[\tau>t \mid \mathcal{F}_{t}\right]
$$

chosen to be càdlàg, associated with $\tau$ by Azéma ( $[3]$ ), (note that $Z_{t}>0$ on the set $\{t<\tau\}$ );

- the $\mathbb{F}$ dual projections of the process $\left(\mathbf{1}_{\{\tau \leq \cdot\})}\right.$, namely the dual optional projection denoted $A_{t}^{\tau}$ and the dual predictable projection denoted $a_{t}^{\tau^{-}}$(the definitions of these two notions can be found in the Appendix A);

- the càdlàg martingale

$$
\mu_{t}^{\tau}=\mathbf{E}\left[A_{\infty}^{\tau} \mid \mathcal{F}_{t}\right]=A_{t}^{\tau}+Z_{t}^{\tau} .
$$

- the Doob-Meyer decomposition of (3.1):

$$
Z_{t}^{\tau}=m_{t}^{\tau}-a_{t}^{\tau}
$$

where $m^{\tau}$ is an $\mathbb{F}$-martingale. 
A common assumption in the literature is that the random time $\tau$ avoids the $\mathbb{F}$ stopping times, that is $\mathbf{P}(\tau=$ $T)=0$ for any $\mathbb{F}$ stopping time $T$. In this case, $A^{\tau}=a^{\tau}$ is continuous and $\tau$ is totally inaccessible, a property useful later on.

The Azéma supermartingale $\left(Z_{t}^{\tau}\right)$ is the main tool for computing the $\mathbb{G}$ predictable compensator of $\mathbf{1}_{\{\tau \leq t\}}$ :

Theorem 3.1 ( [26]). The process:

is a $\mathbb{G}$ martingale.

$$
N_{t}:=\mathbf{1}_{\{\tau \leq t\}}-\int_{0}^{t \wedge \tau} \frac{1}{Z_{s-}^{\tau}} d a_{s}^{\tau}
$$

We shall assume in the rest of the paper that:

(H) Every $\mathbb{F}$ (local-)martingale is a $\mathbb{G}$ (local-)martingale. We say that the filtration $\mathbb{F}$ is immersed in $\mathbb{G}$ and denote this property by the symbol: $\mathbb{F} \hookrightarrow \mathbb{G}$.

The immersion property was studied in [5], [14]. This assumption can be related to absence of arbitrages in the market model (see [4], [9]).

It is known that in our framework $(\mathbf{H})$ is equivalent to the following: For all $s \leq t$,

$$
\mathbf{P}\left[\tau \leq s \mid \mathcal{F}_{t}\right]=\mathbf{P}\left[\tau \leq s \mid \mathcal{F}_{\infty}\right]
$$

It follows that under $(\mathbf{H})$, the Azéma supermartingale is a decreasing process, i.e.,

$$
Z^{\tau}=1-A^{\tau}
$$

Moreover, when the immersion property holds, simple projection formulas for stochastic integrals hold:

Proposition 3.2 ( [5]). Suppose that $\mathbb{F} \hookrightarrow \mathbb{G}$.

(i) Let $M$ be an $\mathbb{F}$ local martingale and $H$ a $\mathbb{G}$ bounded process. Then the $\mathbb{F}$ optional projection of the process $\left(\int H d M\right)$ is given by $\int{ }^{o} H d M$, where ${ }^{o} H$ is the $\mathbb{F}$ optional projection of $H$.

(ii) If $M$ is a $\mathbb{G}$ square integrable martingale and $H$ an $\mathbb{F}$ bounded process. Then the $\mathbb{F}$ optional projection of the process $\left(\int H d M\right)$ is given by $\int H d^{\circ} M$, where ${ }^{o} M$ is the $\mathbb{F}$ optional projection of $M$.

\section{Decompositions of A Stopping time}

We now investigate some general properties of a stopping time $\tau$ of a filtration $\mathbb{G}$ constructed by progressively enlarging a filtration $\mathbb{F}$, as explained in Section 3. We recall that we assume that $\tau<\infty$ a.s.. We begin with a financial example and then show a general construction.

Suppose that $T^{k}, k \in N^{*} \subset \mathbb{N}^{*}$ are unexpected times when negative shocks occur in the economy. They are finite, totally inaccessible stopping times in the filtration $\mathbb{F}$. Morover $\mathbf{P}\left(T^{i}=T^{j}\right)=0, i \neq j$. Now, we suppose that a firm's financial health is affected by these shocks, and this impact may be so severe that can trigger the default of the company i.e.:

$$
\mathbf{P}\left(\tau=T^{k}\right)>0, \forall k \in N^{*}
$$

Denote: $p_{t}^{k}:=\mathbf{P}\left(\tau=T^{k} \mid \mathcal{F}_{t}\right)$ such that we obtain:

$$
\sum_{k \in N^{*}} p_{t}^{k} \leq 1
$$

Lemma 4.1. (a) If $\sum_{k \in N^{*}} p_{0}^{k}=1$ then $\tau$ is a $\mathbb{G}$ totally inaccessible stopping time.

(b) If $\tau$ is a $\mathbb{G}$ totally inaccessible stopping time and $\sum_{k \in N^{*}} p_{0}^{k}<1$, then there exists a $\mathbb{G}$ totally inaccessible stopping time $T^{0}$ such that:

$$
\tau=\sum_{i \in N} T^{i} \mathbf{1}_{\left\{T^{i}=\tau\right\}} .
$$

where $N=N^{*} \cup\{0\}$ and hence $\sum_{k \in N} p_{t}^{k}=1$, where $p_{t}^{0}=\mathbf{P}\left(\tau=T^{0} \mid \mathcal{F}_{t}\right)$. 
Proof. (a) Follows from the definition of a totally inaccessible stopping time.

(b) Denote:

$$
T^{0}:=\tau \mathbf{1}_{\left\{\tau \neq T^{i}, \forall i \in N^{*}\right\}}+\infty \mathbf{1}_{\left\{\exists i \in N^{*}, \tau=T^{i}\right\}}
$$

Obviously $T^{0}$ is a $\mathbb{G}$ stopping time: $\left\{T^{i}=\tau\right\} \in \mathcal{G}_{\tau}$ for $i \in N^{*}$, and therefore $\left\{T^{0}<t\right\}=\{\tau<t\} \bigcap_{i \in N}\left\{T^{i}=\right.$ $\tau\}^{c}$ is in $\mathcal{G}_{t}$. Let $S$ be any predictable $\mathbb{G}$ stopping time. Then, $\mathbf{P}\left(T^{0}=S<\infty\right)=\mathbf{P}(\{\tau=S<\infty\} \cup\{\tau \neq$ $\left.\left.T^{i}, \forall i \in N^{*}\right\}\right) \leq \mathbf{P}(\tau=S<\infty)=0$, since $\tau$ is totally inaccessible.

Remark. Without loss of generality, we may assume that $T^{0}$ avoids all $\mathbb{F}$ finite stopping times, that is:

$$
\mathbf{P}\left(T^{0}=T<\infty\right)=0 \text { for any } \mathbb{F} \text { stopping time } T, T<\infty \text { a.s.. }
$$

The justification is as follows. Let us denote by $\mathcal{H}$ the set of all elements of $\mathcal{F}$ that are of the form $\left(\cup_{\alpha}\left\{T^{\alpha}=T^{0}<\infty\right\}\right)$ where $\left(T^{\alpha}\right)$ is a countable sequence of $\mathbb{F}$ stopping times. The elements of the set $\mathcal{H}$ belong to $\mathcal{G}_{T^{0}}$ (see [12], T29, page 53). $\mathcal{H}$ is stable under countable unions and hence ess sup $\mathcal{H} \in \mathcal{G}_{T^{0}}$. Let $H$ be any element of ess sup $\mathcal{H}$. We define $\bar{T}^{0}$ as the restriction of $T^{0}$ to the complement of $H$, namely:

$$
\bar{T}^{0}:=T^{0} \mathbf{1}_{H^{c}}+\infty \mathbf{1}_{H}
$$

We have that $\bar{T}^{0}$ is still a stopping time for the filtration $\mathbb{G}$, since $H \in \mathcal{G}_{T^{0}}$. Furthermore, by construction, $\bar{T}^{0}$ avoids all $\mathbb{F}$-stopping times. We consider an exhausting sequence of $H$ that we denote $\left(T^{\alpha}\right)_{\alpha \in M}$, i.e., $\left\{\omega \mid T^{\alpha}(\omega)=T^{\beta}(\omega)\right\}=\emptyset$ for all $\alpha \neq \beta, \alpha, \beta \in M$ (an exhausting sequence exists, because by definition $H$ is a thin set).

From (4.1), we also have that $\left\{\omega \mid T^{\alpha}(\omega)=T^{i}(\omega)\right\}=\emptyset$ for any $\alpha \in M$ and $i \in N^{*}$.

Then, the times $T^{i}, i \in N^{*} \cup M$ can be considered times of economic shocks and $\bar{T}^{0}$ is $\mathbb{G}$ totally inaccessible and avoids all finite $\mathbb{F}$ stopping times. Also $\tau=\sum_{i \in N^{*} \cup N} T^{i} \mathbf{1}_{\left\{T^{i}=\tau\right\}}+\bar{T}^{0} \mathbf{1}_{\left\{\bar{T}^{0}=\tau\right\}}$.

We now show that it is natural to construct default models as above, since any stopping time $\tau$ admits decompositions involving sequences of $\mathbb{F}$ stopping times as follows:

Proposition 4.2. There exists a sequence $\left(T^{i}\right)_{i \geq 1}$, of $\mathbb{F}$ stopping times, such that

$$
\mathbf{P}\left(T^{i}=T^{j}<\infty\right)=0 \quad i \neq j
$$

and

$$
\mathbf{P}\left(\tau=T^{i}\right)>0 \text { whenever } \mathbf{P}\left(T^{i}<\infty\right)>0
$$

and a totally inaccessible $\mathbb{G}$ stopping time $T^{0}$ such that $T^{0}$ avoids all finite $\mathbb{F}$ stopping times and such that:

$$
\tau=\sum_{i \geq 0} T^{i} \mathbf{1}_{\left\{T^{i}=\tau\right\}}
$$

The $\mathbb{G}$ stopping time $\tau$ is totally inaccessible if and only if the $\mathbb{F}$ stopping times $\left(T^{i}\right)_{i \geq 1}$ are totally inaccessible.

Proof. We begin with a useful lemma:

Lemma 4.3. Let $T$ be an $\mathbb{F}$ stopping time. $\mathbf{P}(T=\tau)>0$ if and only if the event $\left\{A_{T}^{\tau}(\omega) \neq A_{T-}^{\tau}(\omega)\right\}$ has a strictly positive probability (one says that $A^{\tau}$ charges $T$ ). 
Proof. Let us recall that $A^{\tau}$ is the dual optional projection of the increasing process $H_{t}:=\mathbf{1}_{\{\tau \leq t\}}$, which has a unique jump of size 1 at the time $\tau$. Therefore, for any stopping time $T$ we have: $\Delta H_{T}=\mathbf{1}_{\{\tau=T\}}$. If $T$ is an $\mathbb{F}$ stopping time, then by Theorem VI.76. in [15]:

$$
\Delta A_{T}^{\tau}=\mathbf{E}\left[\Delta H_{T} \mid \mathcal{F}_{T}\right]=\mathbf{P}\left(\tau=T \mid \mathcal{F}_{T}\right) .
$$

Recall that $A^{\tau}$ is increasing, hence $\Delta A^{\tau}$ is nonnegative. The result follows.

We now prove the proposition. There are two possible situations:

1. $A^{\tau}$ continuous. This corresponds to the situation when $\tau$ avoids all $\mathbb{F}$ stopping times. Therefore the decomposition (4.5) holds with all $\left(T^{i}\right)_{i>1}$ infinite a.s. and $T^{0}=\tau$ a.s.

2. $A^{\tau}$ discontinuous. Let $T^{i}, i \geq 1$ be the jump times of $A^{\tau}$. If there are finitely many, we simply set the remaining stopping times in the sequence to be infinite (notice that (4.3) is satisfied by this sequence). We obtain from Lemma 4.3 that:

(i) $\mathbf{P}\left(\tau=T^{i}\right)>0$ whenever $\mathbf{P}\left(T^{i}<\infty\right)>0$;

(ii) $\tau$ avoids any $\mathbb{F}$ stopping time with the graph disjoint of the union of the graphs of $T^{i}, i \geq 1$.

We define $T^{0}$ as the restriction of $\tau$ to the set $E=\left(\cup_{i \geq 1}\left\{T^{i}=\tau\right\}\right)^{c}$. $T^{0}$ is a $\mathbb{G}$ stopping time since $E \in \mathcal{G}_{\tau}$. By construction, $T^{0}$ satisfies,

$$
\mathbf{P}\left(T^{0}=T^{i}<\infty\right)=0 \quad \forall i \geq 1 .
$$

From this and $(i i)$ it follows that $T^{0}$ avoids all finite $\mathbb{F}$-stopping times. Hence, $T^{0}$ is indeed totally inaccessible. Notice that if $\mathbf{P}(E)=0$ then $T^{0}=\infty$ avoids the finite $\mathbb{F}$ stopping times and is totally inaccessible ( $\infty$ is the only time which is both accessible and totally inaccessible).

The last statement in the proposition follows from the definition of a totally inaccessible stopping time.

Remark. From the above proof, we see that we can in fact choose $\left(T^{i}\right)_{i \geq 1}$ to be any sequence that exhausts the jump times of the process $\left(A_{t}^{\tau}\right)$ and therefore the sequence is not uniquely defined. Useful later on will be to notice that the sequence $\left(T^{i}\right)_{i \geq 1}$ can be chosen such that it contains only totally inaccessible and predictable $\mathbb{F}$ stopping times (indeed, one can decompose a stopping time first in its accessible and totally inaccessible part, and then the accessible part in a sequence of predictable times). On the other hand, the time $T^{0}$ can be uniquely defined (up to null sets) if chosen to be infinite on the set $\left\{\tau=T^{i}, i \geq 1\right\}$, as in the construction in equation (4.2) with $N^{*}=\mathbb{N}^{*}$.

Now, we provide an economic interpretation of the sequence $\left(T^{i}\right)$, when they are totally inaccessible stopping times. As the filtration $\mathbb{F}$ is generated by the prices of default-free claims, there exist some default free assets which have jumps at the $\mathbb{F}$ stopping times $\left(T^{i}\right)_{i \in \mathbb{N}^{*}}$ which are not infinite. Therefore, when the default arrives at one of these times, some default-free asset prices react abruptly by jumps. The interpretation is that the default $\tau$ either has a macroeconomic impact, or is triggered by some macroeconomic shock. On the opposite, since $T^{0}$ avoids all finite $\mathbb{F}$-stopping times, when default arrives at this time (i.e., on the set $\left\{\tau=T^{0}\right\}$ ), there will be no impact of the default event on the prices of the default-free assets, that is no default-free asset price will jump. Therefore, we propose the following definition in a default modeling context:

Definition 4.4. Let $\tau$ be a default time which is a totally inaccessible $\mathbb{G}$ stopping time. Take a decomposition of $\tau$ as in (4.5). Then, we shall call $T^{i}, i \in \mathbb{N}^{*}$ times of macroeconomic shocks, and $T^{0}$ the idiosincratic default time (i.e. when the default is caused by the unique and specific circumstances of the company, as opposed to the overall market circumstances).

In the remaining of this section, starting from some given characteristics of a decomposing sequence of times $\left(T^{i}\right)_{i \in \mathbb{N}}$, we shall derive the useful properties of $\tau$, namely the compensator (Proposition 4.5) and the Azéma supermartingale (Proposition 4.10). $\tau$ and the sequence $\left(T^{i}\right)_{i \in \mathbb{N}}$ will be always supposed to fulfill the properties 
stated in Proposition 4.2 (except $T^{0}$, they are not necessarily totally inaccessible). Moreover, we suppose $T^{0}$ to be infinite on the set $\left\{\tau \neq T^{0}\right\}$.

Let us denote by $\left(\Lambda_{T^{i} \wedge t}^{i}\right)$ the $\mathbb{F}$-compensators of the $\mathbb{F}$ stopping times $T^{i}, i \in \mathbb{N}^{*}$. It follows that $\left(\Lambda_{t \wedge T^{i}}^{i}\right)$, $i \in \mathbb{N}^{*}$ are $\mathbb{F}$ adapted and since $\mathbb{F} \hookrightarrow \mathbb{G}$ they are also the $\mathbb{G}$ compensators of $T^{i}, i \in \mathbb{N}^{*}$. We introduce the $\mathbb{F}$ (and $\mathbb{G}$ )-martingales:

$$
N_{t}^{i}:=\mathbf{1}_{\left\{T^{i} \leq t\right\}}-\Lambda_{t \wedge T^{i}}^{i}, \text { for } i \in \mathbb{N}^{*} .
$$

On the other hand, the time $T^{0}$ is not an $\mathbb{F}$ stopping time. We denote $a_{t}^{0}$ the $\mathbb{F}$ dual optional projection of $\mathbf{1}_{\left\{T^{0} \leq \cdot\right\}}$. Since $T^{0}$ avoids all finite $\mathbb{F}$ stopping times, $\left(a_{t}^{0}\right)_{t \in[0, \infty)}$ is continuous. Let us introduce $\tilde{\mathcal{F}}_{t}:=$ $\mathcal{F}_{t} \vee \sigma\left(t \wedge T^{0}\right)$ and notice that $\tilde{\mathcal{F}}_{t} \subset \mathcal{G}_{t}, t \geq 0$. Since $\mathbb{F} \hookrightarrow \mathbb{G}$ it follows that $\mathbb{F} \hookrightarrow \tilde{\mathbb{F}} \subset \mathbb{G}$, hence the Azéma supermartingale of the time $T^{0}$ is decreasing (see equation (3.4)) and equals

$$
Z_{t}^{0}:=\mathbf{P}\left(T^{0}>t \mid \mathcal{F}_{t}\right)=1-a_{t}^{0} .
$$

Notice that $\lim _{t \rightarrow \infty} Z_{t}^{0}=\mathbf{P}\left(T^{0}=\infty \mid \mathcal{F}_{\infty}\right)$. Let us also denote $\left(\Lambda_{t}^{0}\right)$ the $\mathbb{G}$-compensator of $T^{0}$, such that:

$$
N_{t}^{0}:=\mathbf{1}_{\left\{T^{0} \leq t\right\}}-\Lambda_{t}^{0}, \quad t \geq 0
$$

is a $\mathbb{G}$-martingale.

Now, we give the decomposition of the compensator of $\tau$ given those of the times $\left(T^{i}\right)$.

Proposition 4.5. Let $g_{t}^{i}:=\mathbf{P}\left(\tau=T^{i} \mid \mathcal{G}_{t}\right)$ for $t \geq 0$ and $i \in \mathbb{N}$. The $\mathbb{G}$-predictable compensator of $\mathbf{1}_{\{\tau \leq \cdot\}}$ denoted $\left(\Lambda_{t \wedge \tau}\right)$ satisfies:

$$
\Lambda_{t \wedge \tau}=\sum_{i \geq 1} \int_{0}^{t}\left(g_{s-}^{i}+u_{s}^{i}\right) d \Lambda_{s \wedge T^{i}}^{i}+\Lambda_{T^{0} \wedge t}^{0},
$$

where for $i \geq 1,\left(u_{t}^{i}\right)$ is a predictable process that satisfies $\left\langle g^{i}, N^{i}\right\rangle_{t}=\int_{0}^{t} u_{s}^{i} d \Lambda_{T^{i} \wedge s}^{i}$. Hence, the $\mathbb{G}$-martingale $N_{t}=\mathbf{1}_{\{\tau \leq t\}}-\Lambda_{t \wedge \tau}$ decomposes as:

$$
N_{t}=\sum_{i \geq 1}\left(\int_{0}^{t}\left(g_{s-}^{i}+u_{s}^{i}\right) d N_{s}^{i}+\left(\Delta g_{T^{i}}^{i}-u_{T^{i}}^{i}\right) \mathbf{1}_{\left\{T^{i} \leq t\right\}}\right)+N_{t}^{0} .
$$

Therefore $\tau$ has a $\mathbb{G}$ intensity $\lambda$, i.e., $\Lambda_{t \wedge \tau}=\int_{0}^{t \wedge \tau} \lambda_{s} d s$ if and only if $\mathbb{G}$ intensities exist on $[0, \infty)$ for the times $T^{i}, i \geq 0$. Then, denoting by $\lambda^{i}$ the $\mathbb{G}$ intensity of $T^{i}$, the following relation holds:

$$
\lambda_{t}=\sum_{i \geq 1}\left(g_{t}^{i}+u_{s}^{i}\right) \lambda_{t}^{i} \mathbf{1}_{\left\{T^{i}>t\right\}}+\lambda_{t}^{0} .
$$

Proof. We first establish the expression (4.7) of the process $\left(\Lambda_{t \wedge \tau}\right)$. The result follows from:

$$
\mathbf{1}_{\{\tau \leq t\}}=\sum_{i \geq 0} \mathbf{1}_{\left\{T^{i}=\tau\right\}} \mathbf{1}_{\left\{T^{i} \leq t\right\}}=\sum_{i \geq 1} g_{T^{i}}^{i} \mathbf{1}_{\left\{T^{i} \leq t\right\}}+\mathbf{1}_{\left\{T^{0} \leq t\right\}} .
$$

For obtaining the last equality above, we used for $i \geq 1$ the equality $g_{T^{i}}^{i}=\mathbf{1}_{\left\{T^{i}=\tau\right\}}$ and for $i=0,\left\{T^{0} \leq t\right\} \subset$ $\left\{\tau=T^{0}\right\}$ (recall that $T^{0}$ is assumed to be infinite on the set $\left\{\tau \neq T^{0}\right\}$ ).

In the remaining of the proof, we denote $X^{(\mathbb{G}, p)}$ the dual predictable projection of $X$ in the filtration $\mathbb{G}$. We have $\Lambda_{t \wedge \tau}=\left(\mathbf{1}_{\{\tau \leq t\}}\right)^{(\mathbb{G}, p)}$ and we make use of the decomposition in (4.9). By dominated convergence $\left(\sum_{i \geq 1} g_{T^{i}}^{i} \mathbf{1}_{\left\{T^{i} \leq t\right\}}\right)^{(\mathbb{G}, p)}=\sum_{i \geq 1}\left(g_{T^{i}}^{i} \mathbf{1}_{\left\{T^{i} \leq t\right\}}\right)^{(\mathbb{G}, p)}$. We notice that:

$$
g_{T^{i}}^{i} \mathbf{1}_{\left\{T^{i} \leq t\right\}}=\int_{0}^{t} g_{s-}^{i} d \mathbf{1}_{\left\{T^{i} \leq s\right\}}+\Delta g_{T^{i}}^{i} \mathbf{1}_{\left\{T^{i} \leq t\right\}}
$$


For $i \geq 1$, there exists a $\mathbb{G}$-predictable process $\left(u_{t}^{i}\right)$ such that $\left\langle g^{i}, N^{i}\right\rangle_{t}=\int_{0}^{t} u_{s}^{i} d \Lambda_{T^{i} \wedge s}^{i}$ (see [13]). We have that $\left(\int g_{s-}^{i} d \mathbf{1}_{\left\{T^{i} \leq \cdot\right\}}\right)^{(\mathbb{G}, p)}=\int g_{s-}^{i} d \Lambda_{T^{i} \wedge \cdot}^{i}$, therefore we only need to show the equality: $\left(\Delta g_{T^{i}}^{i} \mathbf{1}_{\left\{T^{i} \leq t\right\}}\right)^{(\mathbb{G}, p)}=$ $\left\langle g^{i}, N^{i}\right\rangle_{t}$. As we have remarked previously (see the Remark after Proposition 4.2) it is sufficient to consider the cases when $T^{i}$ is either totally inaccessible or predictable. If $T^{i}$ is totally inaccessible, then $\Delta g_{T^{i}}^{i} \mathbf{1}_{\left\{T^{i} \leq t\right\}}=$ $\left[g^{i}, N^{i}\right]_{t}$ since $N^{i}$ is of finite variation and on $\left\{T^{i} \leq t\right\}$ has one jump of size 1 at $T^{i}$, so that the equality is correct. If $T^{i}$ is predictable, then $\left(\Delta g_{T^{i}}^{i} \mathbf{1}_{\left\{T^{i} \leq t\right\}}\right)^{(\mathbb{G}, p)}=0$ by the predictable stopping theorem. On the other hand $N^{i} \equiv 0,\left\langle g^{i}, N^{i}\right\rangle_{t}=0$ and again the needed equality is correct. Summarizing, we have for $i \geq 1$ :

$$
\left(g_{T^{i}}^{i} \mathbf{1}_{\left\{T^{i} \leq t\right\}}\right)^{(\mathbb{G}, p)}=\int_{0}^{\cdot}\left(g_{s-}^{i}+u_{s}^{i}\right) d \Lambda_{s \wedge T^{i}}^{i} .
$$

Hence, the expression of the compensator $\Lambda$ is proved; we see that it is a sum of increasing processes (indeed each process $g_{T^{i}}^{i} \mathbf{1}_{\left\{T^{i} \leq \cdot\right\}}$ being increasing, so is its $\mathbb{G}$ dual predictable projection). By definition, the $\mathbb{G}$ stopping time $\tau$ admits a $\mathbb{G}$ intensity if and only if the compensator process $\Lambda$ is absolutely continuous with respect to the Lebesgue measure, that is, there exists a nonnegative, $\mathbb{G}$ predictable process $\lambda$ such that $\Lambda_{t \wedge \tau}=\int_{0}^{t \wedge \tau} \lambda_{s} d s$. This is the case if and only if each component in the sum in the expression (4.7) is absolutely continuous with respect to the Lebesgue measure, that is $\mathbb{G}$ intensities exist for the times $T^{i}, i \geq 0$.

Remark. Some simplifications occur in the case where the filtration $\mathbb{G}$ is quasi right continuous. In this situation $\left\{\tau=T^{i}\right\}$ is $\mathcal{G}_{T^{i}}$ measurable for all $i \geq 0$. Hence $\Delta g_{T^{i}}^{i}$ is $\mathcal{G}_{T^{i}}$. measurable and we have $\Delta g_{T^{i}}^{i}=u_{T^{i}}^{i}$ (and this quantity is null in the case where $T^{i}$ is predictable).

We now illustrate with two simple examples the above proposition, the aim being to gain some intuition about the processes involved in Proposition 4.5 (the examples are indeed so simple that the formulas could also be obtained more directly).

Example 4.6. Let us suppose that $\tau=T^{1} \wedge T^{2}$ where $T^{1}$ is an exponential time with parameter $\alpha$ and $T^{2}$ is an exponential time with parameter $\gamma$. It is easy to check that:

$$
g_{t}^{1}=\frac{\alpha}{\alpha+\gamma}+\frac{\gamma}{\alpha+\gamma} N_{t \wedge T^{2}}^{1}-\frac{\alpha}{\alpha+\gamma} N_{t \wedge T^{1}}^{2}
$$

(indeed this is a martingale and $g_{T^{1}}^{1}=\mathbf{1}_{\left\{T^{1}<T^{2}\right\}}$ ). Therefore, $g_{t-}^{1}=\frac{\alpha}{\alpha+\gamma}$ on $[0, \tau]$. Also, $\left\langle g^{1}, N^{1}\right\rangle_{t}=\frac{\alpha \gamma}{\alpha+\gamma}(t \wedge \tau)$, hence $u_{t}^{1}=\frac{\gamma}{\alpha+\gamma}$. Similarly:

$$
g_{t}^{2}=\frac{\gamma}{\alpha+\gamma}+\frac{\alpha}{\alpha+\gamma} N_{t \wedge T^{1}}^{2}-\frac{\gamma}{\alpha+\gamma} N_{t \wedge T^{2}}^{1},
$$

$g_{t-}^{2}=\frac{\gamma}{\alpha+\gamma}$ on $[0, \tau],\left\langle g^{2}, N^{2}\right\rangle_{t}=\frac{\alpha \gamma}{\alpha+\gamma}(t \wedge \tau)$, hence $u_{t}^{2}=\frac{\alpha}{\alpha+\gamma}$. Using the above proposition, we obtain that the compensator of $\tau$ is (as expected):

$$
\Lambda_{t \wedge \tau}=\int_{0}^{t \wedge \tau}\left(g_{s-}^{1}+u_{s}^{1}\right) \alpha d s+\int_{0}^{t \wedge \tau}\left(g_{s-}^{2}+u_{s}^{2}\right) \gamma d s=(\alpha+\gamma)(t \wedge \tau) .
$$

Example 4.7. As in the example above, let us suppose that $T^{1}$ is an exponential time with parameter $\alpha$ and $T^{2}$ is an exponential time with parameter $\gamma$. Let $b$ be a Bernoulli random variable independent of the filtration $\mathbb{F}$ with $\mathbf{P}(b=1)=p$ and $\tau=T^{1} \mathbf{1}_{\{b=1\}}+T^{2} \mathbf{1}_{\{b=0\}}$. Then, $b$ is $\mathcal{G}_{T^{1} \wedge T^{2}}$-measurable and:

$$
\begin{array}{rr}
g_{t}^{1}=p+(b-p) \mathbf{1}_{\left\{t \geq T^{1} \wedge T^{2}\right\}} & u_{t}^{1}=0 \\
g_{t}^{2}=1-p+(p-b) \mathbf{1}_{\left\{t \geq T^{1} \wedge T^{2}\right\}} & u_{t}^{2}=0
\end{array}
$$

therefore:

$$
\Lambda_{t \wedge \tau}=(p \alpha+(1-p) \gamma)\left(t \wedge T^{1} \wedge T^{2}\right)+\mathbf{1}_{\left\{\tau>T^{1} \wedge T^{2}\right\}}(b \alpha+(1-b) \gamma)\left(t \wedge \tau-\left(t \wedge T^{1} \wedge T^{2}\right) .\right.
$$


In the example above, if we suppose that $T^{2}$ is not an $\mathbb{F}$-stopping time, we can define $T^{0}=T^{2} \mathbf{1}_{\{b=0\}}+$ $\infty \mathbf{1}_{\{b=1\}}$. Then, in order to apply Proposition 4.5, one needs an expression for $\Lambda^{0}$.

Below, the general expression of $\Lambda^{0}$ is given, that uses some $\mathbb{F}$-adapted processes, in particular the Azéma supermartingale of $\tau$ :

Lemma 4.8. The $\mathbb{G}$ compensator of $T^{0}$ is given by:

$$
\Lambda_{t}^{0}:=\int_{0}^{t \wedge \tau} \frac{1}{Z_{s-}^{\tau}} d a_{s}^{0}, \quad t \in[0, \infty)
$$

and $\Lambda_{\infty}^{0}=\Lambda_{\tau}^{0} \mathbf{1}_{\left\{T^{0}<\infty\right\}}+\mathbf{1}_{\left\{T^{0}=\infty\right\}}$.

Proof. Notice that the process $\Lambda_{t}^{0}=\int_{0}^{t \wedge \tau} \frac{d a_{s}^{0}}{Z_{s-}^{\tau}}+\left(1-\int_{0}^{\tau} \frac{d a_{s}^{0}}{Z_{s-}^{\tau}}\right) \mathbf{1}_{\left\{T^{0}=\infty\right\}} \mathbf{1}_{\{t=\infty\}}$ is predictable, since it is continuous on $[0, \infty)$ with a possible jump to 1 at $t=\infty$. We need to show that for any bounded, $\mathbb{G}$-predictable process $H$ we have:

$$
\mathbf{E}\left[H_{T^{0}}\right]=\mathbf{E}\left[\int_{0}^{\infty} H_{s} d \Lambda_{s}^{0}\right] .
$$

Since $T^{0}$ may be infinite, the behavior of predictable processes $H$ at $t=\infty$ is playing a role.

Every predictable process $H$ can be decomposed as a sum of two predictable processes: $H=H^{1}+H^{2}$, where $H_{\infty}^{1}=0$ a.s. and $H^{2}$ is of the form $H_{t}^{2}=H_{t} \mathbf{1}_{t=\infty}$. Therefore, it is sufficient to prove the equality (4.10) for processes of the form $H^{1}$ and $H^{2}$.

First, let us suppose that $H_{\infty}=0$ a.s.. There exists an $\mathbb{F}$-predictable process, that we denote $h$, such that: $H_{t} \mathbf{1}_{\tau \geq t}=h_{t} \mathbf{1}_{\tau \geq t}$, in particular, $H_{\tau}=h_{\tau}$ (see for instance [26], Lemme 1). We assume that $h_{\infty}=0$ a.s. (this is without loss of generality, since $\tau<\infty$ a.s.). Since $\left\{T^{0}<\infty\right\}=\left\{T^{0}=\tau\right\}$, it follows that we also have: $H_{T^{0}}=H_{T^{0}} \mathbf{1}_{T^{0}<\infty}=h_{T^{0}} \mathbf{1}_{T^{0}<\infty}=h_{T^{0}}$. Then, we can write:

$$
\mathbf{E}\left[H_{T^{0}}\right]=\mathbf{E}\left[h_{T^{0}}\right]=E\left[\int_{0}^{\infty} h_{s} d a_{s}^{0}\right]=E\left[\int_{0}^{\infty} \frac{\mathbf{1}_{\tau \geq s} h_{s}}{Z_{s-}^{\tau}} d a_{s}^{0}\right]=E\left[\int_{0}^{\tau} \frac{H_{s}}{Z_{s-}^{\tau}} d a_{s}^{0}\right]=\mathbf{E}\left[\int_{0}^{\infty} H_{s} d \Lambda_{s}^{0}\right] .
$$

Now, let us consider a predictable process $H$ of the form $H_{t}=H_{t} \mathbf{1}_{t=\infty}$. We obtain:

$$
\mathbf{E}\left[H_{T^{0}}\right]=\mathbf{E}\left[H_{T^{0}} \mathbf{1}_{T^{0}=\infty}\right]=\mathbf{E}\left[\int_{0}^{\infty} H_{s} d \Lambda_{s}^{0}\right]
$$

It remains now to establish the important properties of $\tau$ seen as an $\mathbb{F}$ random time. We begin with a short lemma:

Lemma 4.9. If $\mathbb{F} \hookrightarrow \mathbb{G}$, then for $i \in \mathbb{N}^{*}$, the $\mathbb{F}$-martingales $p_{t}^{i}:=\mathbf{P}\left(\tau=T^{i} \mid \mathcal{F}_{t}\right)$ satisfy $p_{t}^{i}=p_{t \wedge T^{i}}^{i}$.

Proof. Let us point out that if $\mathbb{F} \hookrightarrow \mathbb{G}$ then for any $\mathbb{F}$ stopping time $T$ and $\mathcal{G}_{T}$ measurable random variable $G$ we have:

$$
\mathbf{E}\left[G \mid \mathcal{F}_{\infty}\right]=\mathbf{E}\left[G \mid \mathcal{F}_{T}\right] .
$$

Then, the result follows easily from the fact that $p^{i}$ is the $\mathbb{F}$ optional projection of the $\mathbb{G}$-martingale $g^{i}$ which also satisfies $g_{t}^{i}=g_{t \wedge T^{i}}^{i}$ and from Proposition 3.2:

$$
p_{\infty}^{i}=\mathbf{E}\left[g_{\infty}^{i} \mid \mathcal{F}_{\infty}\right]=\mathbf{E}\left[g_{T^{i}}^{i} \mid \mathcal{F}_{\infty}\right]=\mathbf{E}\left[g_{T^{i}}^{i} \mid \mathcal{F}_{T^{i}}\right]=p_{T^{i}}^{i} .
$$


Proposition 4.10. Suppose that $\mathbb{F} \hookrightarrow \mathbb{G}$. The Azéma supermartingale of $\tau$ is given by:

$$
Z_{t}^{\tau}:=1-\left(\sum_{i \geq 1} p_{T^{i}}^{i} \mathbf{1}_{\left\{T^{i} \leq t\right\}}+a_{t}^{0}\right),
$$

and its Doob-Meyer decomposition is:

$$
Z_{t}^{\tau}=\left(1-\hat{N}_{t}\right)-a_{t}^{\tau}
$$

where:

$$
\begin{gathered}
a_{t}^{\tau}:=\sum_{i \geq 1} \int_{0}^{t}\left(p_{s-}^{i}+v_{s}^{i}\right) d \Lambda_{T^{i} \wedge s}^{i}+a_{t}^{0} \\
\hat{N}_{t}:=\mathbf{E}\left[N_{t} \mid \mathcal{F}_{t}\right]=\sum_{i \geq 1} \int_{0}^{t}\left(p_{s-}^{i}+v_{s}^{i}\right) d N_{s}^{i}+\left(\Delta p_{T^{i}}^{i}-v_{T^{i}}^{i}\right) \mathbf{1}_{\left\{T^{i} \leq t\right\}}
\end{gathered}
$$

and for $i \geq 1, v^{i}$ is an $\mathbb{F}$ predictable processes that satisfies $\left\langle N^{i}, p^{i}\right\rangle=\int v_{s}^{i} d \Lambda_{s \wedge T^{i}}^{i}$.

Proof. $A_{t}^{\tau}=\sum_{i \geq 1} p_{T^{i}}^{i} \mathbf{1}_{\left\{T^{i} \leq t\right\}}+a_{t}^{0}$ is easily computed as the $\mathbb{F}$-optional projection of $\mathbf{1}_{\{\tau \leq \cdot\}}$ expressed as in (4.9). Hence we obtain the formula (4.11) from $Z^{\tau}=1-A^{\tau}$.

By definition $a^{\tau}$ is the $\mathbb{F}$ compensator of the process $A^{\tau}$. Its expression can be found using exactly the same arguments that the ones in the proof of the Proposition 4.5, with $p^{i}$ instead of $g^{i}$ and $v^{i}$ instead of $u^{i}$. Therefore, by definition of $A^{\tau}$ and $a^{\tau}$, we have that $\hat{N}=A^{\tau}-a^{\tau}={ }^{o}(N)$ is an $\mathbb{F}$-martingale.

Notice that when the times $\left(T^{i}\right)_{i \geq 1}$ are totally inaccessible, then the processes $\Lambda^{i}$ and $a^{0}$ are continuous, the process $a^{\tau}$ is indeed continuous as required by the property of $\tau$ being totally inaccessible. Also, the above Proposition makes it clear that when $\tau$ does not avoid all $\mathbb{F}$ stopping times $Z^{\tau}$ is discontinuous and $A^{\tau}$ and $a^{\tau}$ may differ. More exactly:

Corollary 4.11. The dual optional and predictable projections of $\tau$ coincide, that is $A^{\tau}=a^{\tau}$ if and only if $\tau$ avoids all totally inaccessible stopping times.

Also, using Theorem 3.1 we can write the intensity $\lambda$ in (4.8) using $\mathbb{F}$ adapted processes, as:

$$
\lambda_{t}=\sum_{i \geq 1}\left(\frac{p_{t-}^{i}+v_{t}^{i}}{Z_{t}^{\tau}}\right) \lambda_{t}^{i} \mathbf{1}_{\left\{T^{i} \geq t\right\}}+\lambda_{t}^{0} .
$$

Notice that when $\tau$ has an intensity (as it is classical in the reduced-form approach) the times $T^{i}, i \geq 1$ are times of jumps in the intensity of $\tau$. However it is not possible to recover the information contained in the formula (4.13) from a given intensity process $\lambda$, since different Azéma supermartingales can lead to the same intensity process $\lambda$.

Example 4.12. Let us suppose that $T^{1}$ is an exponential time with parameter $\alpha$, which is an $\mathbb{F}$ stopping time. Let $T^{\gamma}$ be an exponential random variable with parameter $\gamma$, independent of $\mathcal{F}_{\infty}$ and $b$ be a Bernoulli random variable also independent of $\mathcal{F}_{\infty}$, with $\mathbf{P}(b=0)=p$. Let $\tau=T^{1} \mathbf{1}_{\{b=1\}}+T^{\gamma} \mathbf{1}_{\{b=0\}}$. We define $T^{0}=T^{\gamma} \mathbf{1}_{\{b=0\}}+\infty \mathbf{1}_{\{b=1\}}$. We obtain that $a^{0}=\mathbf{P}\left(T^{0} \leq t \mid \mathcal{F}_{t}\right)=p\left(1-e^{-\gamma t}\right)$. By Proposition 4.10 we obtain that:

$$
\begin{aligned}
Z_{t}^{\tau} & =1-(1-p) \mathbf{1}_{\left\{T^{1} \leq t\right\}}-p\left(1-e^{-\gamma t}\right) \\
a_{t}^{\tau} & =p\left(1-e^{-\gamma t}\right)+(1-p) \alpha\left(t \wedge T^{1}\right)
\end{aligned}
$$

and $\tau$ has an intensity given by:

$$
\lambda_{t}=\mathbf{1}_{\left\{T^{1}>t\right\}}\left(q_{t} \gamma+\left(1-q_{t}\right) \alpha\right)+\mathbf{1}_{\left\{T^{1} \leq t\right\}} \gamma,
$$


where $q_{t}=p e^{-\gamma t} /\left(1-p+p e^{-\gamma t}\right)$.

\section{A DECOMPOSItion OF THE DEFAUlt EVENT RISK PREMIUM}

We recall that our probability space is $(\Omega, \mathcal{F}, \mathbb{F}, \mathbf{P})$ and $\tau$ is a random time which is not an $\mathbb{F}$ stopping time and $\mathbb{G}$ is the progressively enlarged filtration which make $\tau$ a stopping time. We also continue to assume the following:

(H) $\mathbb{F} \hookrightarrow \mathbb{G}$, that is the filtration $\mathbb{F}$ is immersed in $\mathbb{G}$;

(TI) $\tau$ is a $\mathbb{G}$ stopping time and $\left(T^{i}\right)_{i \in \mathbb{N}}$ is a decomposing sequence of $\tau$, i.e.,

$$
\tau=\sum_{i \geq 0} T^{i} \mathbf{1}_{\left\{T^{i}=\tau\right\}}
$$

where $\left(T^{i}\right)_{i \geq 1}$ are $\mathbb{F}$ stopping times, such that $\mathbf{P}\left(T^{i}=T^{j}<\infty\right)=0$ for $i \neq j$ and $T^{0}$ avoids all finite $\mathbb{F}$ stopping times.

The aim of this section is to provide an analysis of the risk premiums that form in a financial market in anticipation of losses which occur at the default event $\tau$. From this perspective, it can be added in the assumption (TI) that the time $\tau$ is totally inaccessible (this assumption will be added later on). The defaultable claims we are going to analyze are $\mathcal{G}_{T}$ measurable random variables ( $T>0$ constant) that have the specific form:

$$
X=P \mathbf{1}_{\{\tau>T\}}+C_{\tau} \mathbf{1}_{\{\tau \leq T\}},
$$

where we assume that $P$ is a positive square integrable, $\mathcal{F}_{T}$-measurable random variable which represents a single payment which occurs at time $T$ and $\left(C_{t}\right)$ is a positive bounded, $\mathbb{F}$-adapted process. $P$ stands for the promised payment, while the process $C$ models the recovery in case of default (or collateral).

We do not assume the recovery process $C$ to be predictable, as it is common in the usual reduced-form setting, since we want to emphasize possible drops in the value of the collateral when macro-economic shocks arrive, which we believe are important phenomena in the credit markets, and should bear risk premiums. In order to obtain explicit formulas for the risk premiums attached to the jumps of the price process at the macro-economic shock times $\left(T^{i}\right)_{i \geq 1}$, the following projection result will play an important role:

Lemma 5.1 ( [30]). Let $T$ be an $\mathbb{F}$ stopping time and $X$ a square integrable random variable which is $\mathcal{F}_{T}$ measurable. Then, there exists an $\mathbb{F}$ predictable process $\left(x_{t}\right)$ such that $\mathbf{E}\left[X \mid \mathcal{F}_{T-}\right]=x_{T}$. Denote $\xi=X-x_{T}$. The process $\left(\xi \mathbf{1}_{\{T \leq t\}}, t \geq 0\right)$ is an $\mathbb{F}$ square integrable martingale which is orthogonal to any $\mathbb{F}$ square integrable martingale $\left(M_{t}\right)$ that has the property that $M_{T}$ is $\mathcal{F}_{T-\text { measurable. }}$

We therefore assume without loss of generality the particular form for the recovery process:

(R) There exist a sequence of predictable processes $\left(c^{i}\right)_{t \geq 0}, i \in \mathbb{N}^{*}$ and a sequence of random variables $\left(\kappa^{i}\right)_{i \in \mathbb{N}^{*}}$ such that the recovery decomposes as:

$$
C_{t}=\hat{C}_{t}-\sum_{i \geq 1}\left(c_{T^{i}}^{i}+\kappa^{i}\right) \mathbf{1}_{\left\{T^{i} \leq t\right\}}
$$

where the process $\left(\hat{C}_{t}\right)_{t \geq 0}$ does not have discontinuities at the stopping times $T^{i}, i \in \mathbb{N}^{*}$ and the processes $\left(\kappa^{i} \mathbf{1}_{\left\{T^{i} \leq t\right\}}\right)$ are $\mathbb{F}$-martingales.

The decomposition in (R) is easy to obtain for a general recovery process: $C_{t}=\hat{C}_{t}+\sum_{i \geq 1} \Delta C_{T^{i}} \mathbf{1}_{\left\{T^{i} \leq t\right\}}$ and the random variables $\Delta C_{T^{i}}$ are $\mathcal{F}_{T^{i}}$-measurable, $i \geq 1$. If $\Delta C_{T^{i}}$ is independent from $\mathcal{F}_{T^{i}-}$, then $\kappa^{i}=-\Delta C_{T^{i}}$. In general, $c_{T^{i}}^{i}=-\mathbf{E}\left[\Delta C_{T^{i}} \mid \mathcal{F}_{T^{i}-}\right], \kappa^{i}=\mathbf{E}\left[\Delta C_{T^{i}} \mid \mathcal{F}_{T^{i}-}\right]-\Delta C_{T^{i}}$. 
Similarily, the $\mathbb{F}$-martingales $p^{i}, i \geq 1$ admit the decomposition:

$$
p_{t}^{i}=\int_{0}^{t} v_{s}^{i} d N_{s}^{i}+\phi^{i} \mathbf{1}_{\left\{T^{i} \leq t\right\}}+\hat{m}_{t}^{i}
$$

where the processes $v^{i}$ are predictable with $\mathbf{E}\left[\left|v_{T^{i}}^{i}\right|\right]<\infty ; \phi^{i}$ are random variables $\mathcal{F}_{T^{i}}$ measurable with $\mathbf{E}\left[\theta^{i} \mid \mathcal{F}_{T^{i}-}\right]=0$ and $\left(\hat{m}_{t}^{i}\right)$ is an $\mathbb{F}$-martingale orthogonal to $N^{i}$.

Let us also denote $R_{t}=\int_{0}^{t} r_{u} d u$, where $r_{u}$ is the locally risk-fee interest rate. We shall assume that $\mathbf{P}$ is a risk neutral measure. We recall that the arbitrage-free price of a defaultable claim is given by the following conditional expectation:

$$
S(X)_{t}:=e^{R_{t}} \mathbf{E}\left[P e^{-R_{T}} \mathbf{1}_{\{\tau>T\}}+C_{\tau} e^{-R_{\tau}} \mathbf{1}_{\{\tau \leq T\}} \mid \mathcal{G}_{t}\right]
$$

Using the enlargement of filtration framework, pre-default prices can always be expressed in terms of an $\mathbb{F}$-adapted process, via projections on the smaller filtration $\mathbb{F}$ as (see [17], [24], [4]):

$$
\mathbf{1}_{\{\tau>t\}} S(X)_{t}=\mathbf{1}_{\{\tau>t\}} \tilde{S}(X)_{t}
$$

where $\tilde{S}(X)$ is $\mathbb{F}$-adapted, given by:

$$
\tilde{S}(X)_{t}:=\frac{e^{R_{t}}}{Z_{t}^{\tau}} \mathbf{E}\left[P e^{-R_{T}} \mathbf{1}_{\{\tau>T\}}+C_{\tau} e^{-R_{\tau}} \mathbf{1}_{\{\tau \in(t, T]\}} \mid \mathcal{F}_{t}\right]
$$

The process $\tilde{S}(X)$ is always well defined on the stochastic interval $[0, \tau)$. We add the assumption that $Z_{t}^{\tau}>0, \forall t$ so that the pre-default price process is well defined $\forall t$. Therefore, we exclude the situation where $\tau$ is an $\mathbb{F}$ stopping time.

We recall below a well known expression of the pre-default price process (see for instance [17], [4], [24]) which holds in a particular case of our framework:

Proposition 5.2 ([4]). Suppose that the process $C$ is predictable and the process $Z^{\tau}$ is continuous. Then

$$
\tilde{S}(X)_{t}=\frac{e^{R_{t}}}{Z_{t}^{\tau}} \mathbf{E}\left[\int_{t}^{T} C_{u} e^{-\left(R_{u}+\Lambda_{u}\right)} d \Lambda_{u}+P e^{-\left(R_{T}+\Lambda_{T}\right)} \mid \mathcal{F}_{t}\right], \quad t \geq 0
$$

Remark. It is known that when $\mathbb{F} \hookrightarrow \mathbb{G}, Z^{\tau}$ is continuous if and only if $\tau$ avoids the $\mathbb{F}$ stopping times, i.e., in the condition (TI) all $T^{i}=\infty$ for $i \geq 1$. The property of $C$ being predictable implies that in the condition (R) the processes $\left(c^{i}\right)$ as well as the random variables $\kappa^{i}$ are null.

We are now going to generalize the expression of the pre-default price to our setting, synthesized in the assumptions $(\mathbf{H}),(\mathbf{T I})$ and $(\mathbf{R})$ and deduce an expression of the default event risk premium. We shall use the general pricing methodology developed in [10]. tion.

To begin, we clarify what we understand by default event risk premium, by introducing the following defini-

Definition 5.3. Suppose that the pre-default price process $\tilde{S}(X)$ of the claim $X$ introduced in (5.1) has a canonical decomposition under the risk neutral measure $\mathbf{P}$ :

$$
\tilde{S}(X)_{t}=\tilde{S}(X)_{0}+\int_{0}^{t} \tilde{S}(X)_{u} d \nu(X)_{u}+M_{t}
$$

where $\left(\nu(X)_{t}\right), t \geq 0$ is a finite variation, predictable process and $\left(M_{t}\right)$ an $\mathbb{F}$-martingale $M_{0}=0$. We call (cumulated) default event risk premium the process $\pi(X)_{t}=\nu(X)_{t}-R_{t}, 0 \leq t \leq T$. 
Intuitively, the default event risk premium represents the additional net yield an investor can earn from a security as a compensation for the losses arriving at the default time. Indeed, $\int_{0}^{\cdot \wedge \tau} \tilde{S}(X)_{u} d \pi(X)_{u}$ represents the compensator of the jump (in practice a loss) that will occur in the price of the claim $X$ at the default time $\tau$. Hence, for totally inaccessible default times, the (cumulated) default event risk premium is an increasing, continuous process while for a predictable stopping time, this is always null.

Example 5.4. Suppose the process $Z^{\tau}$ is continuous and $C$ is predictable as in Proposition 5.2. Then from equation (5.5), it can be easily checked that:

$$
\pi(X)_{t}=\Lambda_{t}-\int_{0}^{t} \tilde{C}_{u} d \Lambda_{u}
$$

where $\tilde{C}=C / \tilde{S}(X)$.

Definition 5.3 can be put in relation with the notion of the instantaneous credit spread, i.e., the artificial discounting rate that one would need to apply to the promised payment, in excess to the risk-free rate, in order to make the price of the defaultable claim equal the expected discounted promised payment. The instantaneous credit spread (when it exists) is an $\mathbb{F}$ adapted process $\left(s_{t}\right)$ that satisfies the equality:

$$
\tilde{S}(X)_{t}=\mathbf{E}\left[P e^{\left.-\int_{t}^{T}\left(r_{u}+s_{u}\right) d u\right)} \mid \mathcal{F}_{t}\right]
$$

After noticing that $\tilde{S}(X)_{T}=P$, we find that $S(X)$ decomposes as in (5.6) with $\pi(X)_{t}=\int_{0}^{t} s_{u} d u$. Therefore, the instantaneous credit spread exists only if the default risk premium $\pi(X)$ is absolutely continuous to Lebesgue measure, and in this case $\pi(X)$ can be interpreted as the cumulated instantaneous spreads.

For pricing the defaultable claims, the following $\mathbb{F}$-martingale will play a crucial role (as developed in [10]):

Definition 5.5. We introduce the following exponential local $\mathbb{F}$-martingale:

$$
D_{t}:=\mathcal{E}\left(\int_{0}^{\cdot} \frac{d m_{s}^{\tau}}{Z_{s-}^{\tau}}\right)_{t}=\mathcal{E}\left(-\int_{0}^{\cdot} \frac{d \hat{N}_{s}}{Z_{s-}^{\tau}}\right)_{t}
$$

where the $\mathbb{F}$-martingale $\hat{N}$ was defined in the equality (4.12). If $\left(D_{t}\right)_{0 \leq t \leq T}$ is a square integrable martingale, we define the default-adjusted measure as:

$$
d \mathbf{Q}^{\tau}:=D_{T} \cdot d \mathbf{P} \quad \text { on } \mathcal{F}_{T}
$$

Remark. It is useful to have in mind another equivalent expression of the local martingale $D$, namely:

$$
D_{t}=Z_{t}^{\tau} e^{\Lambda_{t}}
$$

We thus obtain the following proposition:

Proposition 5.6. Suppose that $T^{i}, i \geq 1$ are totally inaccessible stopping times. Furthermore, assume that $\left(D_{t}\right)_{0 \leq t \leq T}$ is a square integrable $\mathbb{F}$-martingale (for instance $\mathbf{E}\left[e^{2 \Lambda_{T}}\right]<\infty$ ). Then, the pre-default price of the defaultable claims is given by:

$$
\tilde{S}_{t}(X)=e^{\tilde{R}_{t}} \mathbf{E}^{\mathbf{Q}^{\tau}}\left[\int_{t}^{T} e^{-\tilde{R}_{u}} C_{u-} d \Lambda_{u}-\sum_{i \geq 1} \int_{t}^{T} e^{-\tilde{R}_{u}} h_{u}^{i} \frac{p_{u-}^{i}+v_{u}^{i}}{Z_{u-}^{\tau}} d \Lambda_{u \wedge T^{i}}^{i}+P e^{-\tilde{R}_{T}} \mid \mathcal{F}_{t}\right] \quad t<T
$$


where:

$$
\begin{aligned}
\tilde{R}_{t} & :=R_{t}+\Lambda_{t}, \\
h_{t}^{i} & :=c_{t}^{i}+\frac{\tilde{\kappa}_{t}^{i}}{p_{t-}^{i}+v_{t}^{i}}
\end{aligned}
$$

and where, for $i \geq 1, \tilde{\kappa}^{i}$ is the predictable process which satisfies $\mathbf{E}\left[\kappa^{i} \phi^{i} \mid \mathcal{F}_{T^{i}-}\right]=\tilde{\kappa}_{T^{i}}^{i}$.

In particular, if the recovery process $C$ does not have discontinuities at the stopping times $T^{i}, i \geq 1$, then the pre-default price of the defaultable claims is given by:

$$
\tilde{S}_{t}(X)=e^{\tilde{R}_{t}} \mathbf{E}^{\mathbf{Q}^{\tau}}\left[\int_{t}^{T} C_{u} e^{-\tilde{R}_{u}} d \Lambda_{u}+P e^{-\tilde{R}_{T}} \mid \mathcal{F}_{t}\right] \quad t<T
$$

Proof. The pricing formulas can be derived using arguments similar to those in [10] (Proposition 4.3.), where $C$ was supposed predictable and $\Lambda$ continuous. We shall extend the formulas to hold for more general $C$ and $\Lambda$ (however in that paper, the (H) hypothesis was not necessarily holding).

First, let us notice from (5.4) that:

$$
\tilde{S}(P)_{t}=\frac{e^{R_{t}}}{Z_{t}^{\tau}} \mathbf{E}\left[P e^{-R_{T}} Z_{T}^{\tau} \mid \mathcal{F}_{t}\right]=\frac{e^{\tilde{R}_{t}}}{D_{t}} \mathbf{E}\left[P e^{-\tilde{R}_{T}} D_{T} \mid \mathcal{F}_{t}\right]=e^{\tilde{R}_{t}} \mathbf{E}^{\mathbf{Q}^{\tau}}\left[P e^{-\tilde{R}_{T}} \mid \mathcal{F}_{t}\right]
$$

By linearity of the conditional expectation, we only need to show the equality:

$$
\tilde{S}\left(C_{\tau} \mathbf{1}_{\{\tau \leq T\}}\right)_{t}=e^{\tilde{R}_{t}} \mathbf{E}^{\mathbf{Q}^{\tau}}\left[\int_{t}^{T} e^{-\tilde{R}_{u}} C_{u-} d \Lambda_{u}-\sum_{i \geq 1} \int_{t}^{T} e^{-\tilde{R}_{u}} h_{u}^{i} \frac{p_{u-}^{i}+v_{u}^{i}}{Z_{u-}^{\tau}} d \Lambda_{u \wedge T^{i}}^{i} \mid \mathcal{F}_{t}\right] .
$$

Indeed, using equation (5.4), the price of a claim that pays $C_{\tau}$ at default and zero otherwise is given by:

$$
\begin{aligned}
& \tilde{S}\left(C_{\tau} \mathbf{1}_{\{\tau \leq T\}}\right)_{t}=\frac{e^{R_{t}}}{Z_{t}^{\tau}} \mathbf{E}\left(e^{-R_{\tau}} C_{\tau} \mathbf{1}_{\{\tau \in(t, T]\}} \mid \mathcal{F}_{t}\right)=e^{\tilde{R}_{t}} D_{t}^{-1} \mathbf{E}\left(\int_{t}^{T} e^{-R_{u}} C_{u} d A_{u}^{\tau} \mid \mathcal{F}_{t}\right) \\
& =e^{\tilde{R}_{t}} D_{t}^{-1} \sum_{i \geq 1} \mathbf{E}\left(e^{-R_{T^{i}}} C_{T^{i}} p_{T^{i}}^{i} \mathbf{1}_{\left\{T^{i} \in(t, T]\right\}} \mid \mathcal{F}_{t}\right) \\
& =e^{\tilde{R}_{t}} D_{t}^{-1} \sum_{i \geq 1} \mathbf{E}\left(e^{-R_{T^{i}}}\left\{C_{T^{i}-}-\left(c_{T^{i}}^{i}+\kappa^{i}\right)\right\}\left(p_{T^{i}-}^{i}+v_{T^{i}}^{i}+\phi^{i}\right) \mathbf{1}_{\left\{T^{i} \in(t, T]\right\}} \mid \mathcal{F}_{t}\right) .
\end{aligned}
$$

Notice that (from Lemma 5.1) the following processes: $\left(e^{-R_{T^{i}}}\left(C_{T^{i}-}-c_{T^{i}}^{i}\right) \phi^{i} \mathbf{1}_{\left\{T^{i} \leq t\right\}}\right)$ and $\left(e^{-R_{T^{i}} \kappa^{i}\left(p_{T^{i}-}^{i}+\right.}\right.$ $\left.\left.v_{T^{i}}^{i}\right) \mathbf{1}_{\left\{T^{i} \in(t, T]\right\}}\right)$ are martingales. Therefore we obtain:

$$
\begin{aligned}
\tilde{S}\left(C_{\tau} \mathbf{1}_{\{\tau \leq T\}}\right)_{t} & =e^{\tilde{R}_{t}} D_{t}^{-1} \sum_{i \geq 1} \mathbf{E}\left(\int_{t}^{T} e^{-R_{u}}\left(C_{u-}-h_{u}^{i}\right)\left(p_{u-}^{i}+v_{u}^{i}\right) d \Lambda_{u \wedge T^{i}}^{i} \mid \mathcal{F}_{t}\right) \\
& =e^{\tilde{R}_{t}} D_{t}^{-1} \sum_{i \geq 1} \mathbf{E}\left(\int_{t}^{T} e^{-\tilde{R}_{u}} D_{u}\left(C_{u-}-h_{u}^{i}\right) \frac{\left(p_{u-}^{i}+v_{u}^{i}\right)}{Z_{u-}^{\tau}} d \Lambda_{u \wedge T^{i}}^{i} \mid \mathcal{F}_{t}\right)
\end{aligned}
$$


Denote $H_{t}^{i}:=\int_{0}^{t} e^{-\tilde{R}_{u}}\left(C_{u-}-h_{u}^{i}\right) \frac{\left(p_{u-}^{i}+v_{u}^{i}\right)}{Z_{u-}^{\tau}} d \Lambda_{u \wedge T^{i}}^{i}$. An integration by parts of the products $H^{i} D$ gives $($ recall that $\Lambda^{i}$ are supposed continuous):

$$
D_{T} H_{T}^{i}=D_{t} H_{t}^{i}+\int_{t}^{T} D_{u-} d H_{u}^{i}+\int_{t}^{T} H_{u}^{i} d N_{u}
$$

Since $C, h^{i}$ and $\frac{\left(p_{t-}^{i}+v_{t}^{i}\right)}{Z_{t-}^{\tau}}$ are bounded and $D$ is a square integrable martingale, $H^{i}$ is also bounded and hence $\int H^{i} d D$ is also a square integrable martingale. Consequently:

$$
\mathbf{E}\left[\int_{t}^{T} D_{u-} d H_{u}^{i} \mid \mathcal{F}_{t}\right]=\mathbf{E}\left[D_{T} H_{T}^{i}-D_{t} H_{t}^{i}-\int_{t}^{T} H_{u}^{i} d D_{u} \mid \mathcal{F}_{t}\right]=\mathbf{E}\left[D_{T}\left(H_{T}^{i}-H_{t}^{i}\right) \mid \mathcal{F}_{t}\right]
$$

Therefore the pricing formula simplifies to :

$$
\tilde{S}\left(C_{\tau} \mathbf{1}_{\{\tau \leq T\}}\right)_{t}=-e^{\tilde{R}_{t}} D_{t}^{-1} \mathbf{E}\left(D_{T} \sum_{i \geq 1} \int_{t}^{T} d H_{u}^{i} \mid \mathcal{F}_{t}\right)
$$

which, after a Girsanov transformation, leads to the desired formula.

As a corollary we obtain the following evolution of the pre-default price process (notice that the term $\tilde{h}^{i}$ is a risk premium attached to the sensitivity of the recovery to the economic shock $T^{i}$ ):

Corollary 5.7. Under the assumptions in Proposition 5.6, there exists an $\mathbb{F}$-martingale $\left(M_{t}\right)$ under the measure $\mathbf{P}$ such that the pre-default price process satisfies the following stochastic differential equation:

$$
\frac{d \tilde{S}_{t}(X)_{t}}{\tilde{S}_{t}(X)_{t}}=d \tilde{R}_{t}-\tilde{C}_{t} d \Lambda_{t}+\sum_{i \geq 1} \tilde{h}_{t}^{i} \frac{\left(p_{t-}^{i}+v_{t}^{i}\right)}{Z_{t-}^{\tau}} d \Lambda_{t \wedge T^{i}}^{i}+\sum_{i \geq 1} d L_{t}^{i}+d M_{t}
$$

where:

$$
\begin{aligned}
\tilde{C}_{t} & =C_{t} / \tilde{S}_{t}(X)_{t} \\
\tilde{h}_{t}^{i} & =h_{t}^{i} / \tilde{S}_{t}(X)_{t},
\end{aligned}
$$

and $L^{i}$ is the $\mathbb{F}$-dual predictable projection of the process $\left(\frac{p_{T^{i}}^{i} \Delta M_{T^{i}}}{Z_{T^{i}-}^{\tau}} \mathbf{1}_{\left\{T^{i} \leq \cdot\right\}}\right)$ for all $i \geq 1$.

Proof. From equation (5.7) it follows that there exists a $\left(\mathbb{F}, \mathbf{Q}^{\tau}\right)$-martingale $\tilde{M}$ such that:

$$
\frac{d \tilde{S}_{t}(X)_{t}}{\tilde{S}_{t}(X)_{t}}=d \tilde{R}_{t}-\tilde{C}_{t} d \Lambda_{t}+\sum_{i \geq 1} \tilde{h}_{t}^{i} \frac{p_{t-}^{i}}{Z_{t-}^{\tau}} d \Lambda_{t \wedge T^{i}}^{i}+d \tilde{M}_{t}
$$

Using the Girsanov's theorem $\tilde{M}=M+\sum_{i \geq 1} L^{i}$ where $M$ is a $\mathbf{P}$ martingale.

In practical applications, one can obtain explicit formulas for the default event risk premium by computing explicitly the dual predictable projections appearing in the corollary above. Let us give the general formulation of these expressions. Any square integrable $\mathbb{F}$ martingale $M$ can be decomposed in sum of orthogonal martingales as follows (see [30]):

$$
M_{t}=\sum_{i \geq 1} \int_{0}^{t} f_{s}^{i} d N_{s}^{i}+\sum_{i \geq 1} \theta^{i} \mathbf{1}_{\left\{T^{i} \leq t\right\}}+\hat{M}_{t}
$$


where the processes $f^{i}$ are predictable with $\mathbf{E}\left[\left|f_{T^{i}}^{i}\right|\right]<\infty ; \theta^{i}$ are random variables $\mathcal{F}_{T^{i}}$ measurable with $\mathbf{E}\left[\theta^{i} \mid \mathcal{F}_{T^{i}-}\right]=0$ (i.e., the process $\left(\theta^{i} \mathbf{1}_{\left\{T^{i} \leq t\right\}}\right)$ is a martingale) and $\left(\hat{M}_{t}\right)$ is a martingale orthogonal to any $N^{i}, i \geq 1$.

Theorem 5.8. Suppose that the $\mathbb{F}$-martingale $M$ appearing in (5.9) decomposes as in (5.10) and the martingales $p^{i}, i \geq 1$ decompose as in (5.3).

Then, the default event risk premium has the expression:

$$
\pi(X)_{t}=\int_{0}^{t}\left(1-\tilde{C}_{u}\right) d \Lambda_{u}+\sum_{i \geq 1} \int_{0}^{t}\left(\tilde{h}_{u}^{i}+\varphi_{u}^{i}\right) \frac{\left(p_{u-}^{i}+v_{u}^{i}\right)}{Z_{u-}^{\tau}} d \Lambda_{u \wedge T^{i}}^{i}
$$

where, for $i \geq 1: \varphi_{t}^{i}:=f_{t}^{i}+\frac{\sigma_{t}^{i}}{p_{t-}^{i}+v_{t}^{i}}$ and $\left(\sigma_{t}^{i}\right)$ is the predictable process which satisfies

$$
\mathbf{E}\left[\theta^{i} \phi^{i} \mid \mathcal{F}_{T^{i}-}\right]=\sigma_{T^{i}}^{i} .
$$

Proof. If $M$ has the representation (5.10) then $\Delta M_{T^{i}}=f_{T^{i}}^{i}+\theta^{i}$. Let us denote $n_{t}^{i}=\theta^{i} \mathbf{1}_{\left\{T^{i} \leq t\right\}}$ which we recall is a square integrable martingale orthogonal to square integrable martingales of the form $\int f_{u} d N_{u}^{i}$. Below, we use the notation $(H)^{p}$ for the $\mathbb{F}$-dual predictable projection of a process $H$. We have:

$$
\begin{aligned}
L_{t}^{i} & =\left(\frac{\Delta M_{T^{i}} p_{T^{i}}^{i}}{Z_{T^{i}}^{\tau}} \mathbf{1}_{T^{i} \leq t}\right)^{p}=\left(\frac{f_{T^{i}}^{i} p_{T^{i}}^{i}}{Z_{T^{i}}^{\tau}} \mathbf{1}_{T^{i} \leq t}\right)^{p}+\left(\frac{p_{T^{i}}^{i}}{Z_{T^{i}-}^{\tau}} \theta^{i} \mathbf{1}_{T^{i} \leq t}\right)^{p} \\
& =\int_{0}^{t} \frac{f_{u}^{i}\left(p_{u-}^{i}+v_{u}^{i}\right)}{Z_{u-}^{\tau}} d \Lambda_{u \wedge T^{i}}^{i}+\left(\int_{0}^{t} \frac{p_{u}^{i}}{Z_{u-}^{\tau}} d n_{u}^{i}\right)^{p}
\end{aligned}
$$

We have that $\Delta p_{T^{i}}^{i}=v_{T^{i}}+\phi^{i}$ and hence: $\int_{0}^{t} p_{u}^{i} d n_{u}^{i}=\int_{0}^{t}\left(p_{u-}^{i}+v_{u}^{i}\right) d n_{u}^{i}+\phi^{i} \theta_{t}^{i} \mathbf{1}_{\left\{T^{i} \leq t\right\}}$. The first term in the sum being a martingale, we obtain that $\left(\int_{0}^{t} p_{u}^{i} d n_{u}^{i}\right)^{p}=\left(\phi^{i} \theta_{t}^{i} 1_{\left\{T^{i} \leq t\right\}}\right)^{p}=\int_{0}^{t} \sigma_{u}^{i} d \Lambda_{u \wedge T^{i}}$ (as explained in [13]). The result follows.

It is possible to decompose the default risk premium appearing in (5.11) in an idiosyncratic and a systematic part (which in turn can be decomposed along premiums attached to each macroeconomic shock) as follows:

$$
\pi(X)_{t}=\int_{0}^{t}\left(1-\tilde{C}_{u}\right) \frac{d a_{u}^{0}}{Z_{u-}^{\tau}}+\sum_{i \geq 1} \int_{0}^{t}\left\{1-\tilde{C}_{u}+\left(\tilde{h}_{u}^{i}+\varphi_{u}^{i}\right)\right\} \frac{p_{u-}^{i}+v_{u}}{Z_{u-}^{\tau}} d \Lambda_{u \wedge T^{i}}^{i} .
$$

We see that each possible macroeconomic shock $T^{i}, i \geq 1$ commands a corresponding risk premium for the possible jumps at $T^{i}$ of the recovery process but also of the martingale $M$, which reflects possible losses of the hedging portfolio.

Example 5.9. Let us consider the model from Example 4.12. The pre-default price of a zero-coupon bond with zero recovery (i.e. $X=\mathbf{1}_{\{\tau>T\}}$ ) is given by:

$$
\tilde{S}_{t}=e^{\tilde{R}_{t}} \mathbf{E}^{\mathbf{Q}^{\tau}}\left[e^{-\tilde{R}_{T}} \mid \mathcal{F}_{t}\right],
$$

where $\tilde{R}_{t}=(r+\gamma) t+\int_{0}^{t \wedge T^{1}}\left(1-q_{u}\right)(\alpha-\gamma) d u$, and the change of measure $\mathbf{Q}^{\tau}$ is defined via the density process $D_{t}=\mathcal{E}\left(\int_{0}^{\cdot}-\left(1-q_{u}\right) d N_{u}^{1}\right)_{t}$. Alternatively,

$$
\tilde{S}_{t}=e^{\tilde{R}_{t}-(r+\gamma) T} m_{t}
$$


where the $\mathbf{Q}^{\tau}$-martingale $m$ is given by:

$$
m_{t}=\mathbf{E}^{\mathbf{Q}^{\tau}}\left[e^{-\int_{0}^{T \wedge T^{1}}\left(1-q_{u}\right)(\alpha-\gamma) d u} \mid \mathcal{F}_{t}\right]
$$

Simple (but lengthy) computations show that:

$$
m_{t}=\mathcal{E}\left(\int_{0}^{\cdot} H_{u} d \tilde{N}_{u}^{1}\right)
$$

where $\tilde{N}_{t}^{1}:=\mathbf{1}_{\left\{T^{1} \leq t\right\}}-\int_{0}^{t \wedge T^{1}} \tilde{\alpha}_{u} d u$ with $\tilde{\alpha}_{t}:=\alpha q_{t}$ being the intensity of $T^{1}$ under the measure $\mathbf{Q}^{\tau}$ (hence $\tilde{N}^{1}$ is a $\mathbf{Q}^{\tau}$-martingale), and $H_{t}:=\left(1-e^{-(T-t)(\alpha-\gamma)}\right) /\left((1-p) e^{-(T-t)(\alpha-\gamma)}+p e^{-\gamma t}\right)$. Finally, we get that the bond price dynamics before default and before the maturity is given by:

$$
\begin{aligned}
d \tilde{S}_{t} & =\tilde{S}_{t-}\left(d \tilde{R}_{t}+H_{t} d \tilde{N}_{t}^{1}\right) \\
& =\tilde{S}_{t-}\left(d R_{t}+\left(\lambda_{t}+\mathbf{1}_{\left\{T^{1}>t\right\}} H_{t}\left(1-q_{t}\right) \alpha\right) d t+H_{t} d N_{t}^{1}\right)
\end{aligned}
$$

Hence the default event risk premium in this example is given by:

$$
\pi_{t}=\int_{0}^{t}\left(\lambda_{u}+\mathbf{1}_{\left\{T^{1}>t\right\}} H_{u}\left(1-q_{u}\right) \alpha\right) d u
$$

We can notice that:

(i) if $\gamma<\alpha$ then $\pi_{t}>\int_{0}^{t} \lambda_{u} d u$ (since $H_{t}>0$ for $t<T$ );

(ii) if $\gamma>\alpha$ then $\pi_{t}<\int_{0}^{t} \lambda_{u} d u$ (since $H_{t}<0$ for $t<T$ );

(iii) if $\gamma=\alpha$ then $\pi_{t}=\int_{0}^{t} \lambda_{u} d u$ (since $\left.H_{t} \equiv 0\right)$.

The financial interpretation is as follows. The default time $\tau$ has as intensity process $\left(\lambda_{t}\right)$. The integral $\int_{0}^{t} \lambda_{u} d u$ represents the default event risk premium corresponding to a default time with intensity process $\left(\lambda_{t}\right)$, when additionally $\tau$ avoids all $\mathbb{F}$-stopping times (the standard assumption). By allowing the default to occur at an F-stopping time, our model captures very different default event risk premiums, which can be bigger or smaller than those under the standard assumption, depending on the intensity of the macroeconomic shocks (in this example $\left.T^{1}\right)$.

Example 5.10. Let us consider again the model from Example 4.12. We price now a recovery payment which is not predictable. More precisely, we suppose that the recovery is some constant $\hat{c}$ before the macroeconomic shock $T^{1}$ but can become null at the macroeconomic shock $T^{1}$. More precisely, we assume there is a Bernoulli random variable $\beta$ with $\mathbf{P}(\beta=0)=p^{\beta}$, and which is only revealed at $T^{1}$ : it is $\mathcal{F}_{T^{1}}$ measurable but independent from $\mathcal{F}_{T^{1}-}$ (in particular the filtration $\mathbb{F}$ is not quasi left continuous). We then suppose that $C_{\tau}=\hat{c} \beta$. We can write the recovery in the form of an $\mathbb{F}$-adapted process, and decompose it as in (5.2):

$$
C_{t}=\hat{c}-\left(c^{1}+\kappa^{1}\right) \mathbf{1}_{\left\{T^{1} \leq t\right\}}
$$

with $c^{1}=\hat{c}\left(1-p^{\beta}\right)$ and $\kappa^{1}=\hat{c}\left(\beta-1+p^{\beta}\right)$. Here, because the martingale $\left(p_{t}^{1}=1-p\right)$ is constant, we have that $\tilde{k}^{1} \equiv 0$ (intuitively, this term reflects a covariation between the joint jumps at time $T^{1}$ of $p^{1}$ and the collateral process). Hence, we have $h^{1}=c^{1}$. The valuation formula for the collateral payment upon default writes here 
in the simpler form:

$$
\begin{aligned}
\tilde{S}\left(C_{\tau} \mathbf{1}_{\{\tau \leq T\}}\right)_{t} & =e^{\tilde{R}_{t}} \mathbf{E}^{\mathbf{Q}^{\tau}}\left[\int_{t}^{T} e^{-\tilde{R}_{u}} C_{u-} d \Lambda_{u}-\int_{t}^{T} e^{-\tilde{R}_{u}} h^{1} \frac{p^{1}}{Z_{u-}^{\tau}} d \Lambda_{u \wedge T^{1}}^{1} \mid \mathcal{F}_{t}\right] \\
& =e^{\tilde{R}_{t}} \mathbf{E}^{\mathbf{Q}^{\tau}}\left[\int_{t}^{T^{1} \wedge T} e^{-\tilde{R}_{u}}\left(\hat{c} q_{u} \gamma+\left(\hat{c}-c^{1}\right)\left(1-q_{u}\right) \alpha\right) d u+\int_{T^{1} \wedge T}^{T} e^{-\tilde{R}_{u}}(\hat{c}+\beta) \gamma d u \mid \mathcal{F}_{t}\right]
\end{aligned}
$$

\section{Conclusion}

In this paper we proposed a decomposition of a default time using sequences of stopping times of the reference filtration $\mathbb{F}$. Our aim was to propose a systematic construction of default times but also to show that some of the simplifying assumptions appearing in the literature lead in fact to an misrepresentation of the risks attached to a long position in a defaultable claim, in particular the risks of losses at the default time. We hope that this analysis sheds light on the behavior of the assets at the default announcement (the so-called "jump to default").

\section{A. Some Definitions}

We consider a filtered probability space $\left(\Omega, \mathcal{F},\left(\mathcal{F}_{t}\right)_{t \geq 0}, \mathbf{P}\right)$ that satisfies the usual conditions.

Theorem A.1 (Optional and predictable projections). Let $X$ be a measurable process, positive or bounded. There exists a unique (up to indistinguishability) optional process ${ }^{o} X$ (resp. predictable process ${ }^{p} X$ ) such that:

$$
\mathbb{E}\left[X_{T} \mathbf{1}_{(T<\infty)} \mid \mathcal{F}_{T}\right]={ }^{o} X_{T} \mathbf{1}_{(T<\infty)} \text { a.s. }
$$

for every stopping time $T$ (resp.

$$
\mathbb{E}\left[X_{T} \mathbf{1}_{(T<\infty)} \mid \mathcal{F}_{T-}\right]={ }^{p} X_{T} \mathbf{1}_{(T<\infty)} \text { a.s. }
$$

for every predictable stopping time $T)$. The process ${ }^{o} X$ is called the optional projection of $X$. The process ${ }^{p} X$ is called the predictable projection of $X$.

A stochastic process which is nonnegative and whose path are increasing and càdlàg, but which is not $\mathbb{F}$-adapted is called a raw increasing process.

Definition A.2 (Dual optional and predictable projections). Let $\left(A_{t}\right)_{t>0}$ be an integrable raw increasing process. We call dual optional projection of $A$ the $\left(\mathcal{F}_{t}\right)$-optional increasing process $\left(A_{t}^{o}\right)_{t \geq 0}$ defined by:

$$
\mathbb{E}\left[\int_{0}^{\infty}{ }^{o} X_{s} d A_{s}\right]=\mathbb{E}\left[\int_{0}^{\infty} X_{s} d A_{s}^{o}\right]
$$

for any bounded adapted $\left(X_{t}\right)$. We call dual predictable projection of $A$ the $\left(\mathcal{F}_{t}\right)$-predictable increasing process $\left(A_{t}^{p}\right)_{t \geq 0}$, such that

$$
\mathbb{E}\left[\int_{0}^{\infty}{ }^{p} X_{s} d A_{s}\right]=\mathbb{E}\left[\int_{0}^{\infty} X_{s} d A_{s}^{p}\right]
$$

for every adapted bounded $\left(X_{t}\right)$.

\section{REFERENCES}

[1] A. Aksamit, Choulli, T. And Jeanblanc, M. Decomposition of Random Times and their Classification, arxiv :1605.03905 (2014).

[2] E. I. Altman, B. Brady, A. Resti And A. Sironi: The link between default and recovery rates: Theory, empirical evidence and implications. Journal of Business 78 (6), (2005), 2203-2227. 
[3] J. AzÉma: Quelques applications de la théorie générale des processus I, Invent. Math. 18 (1972) $293-336$.

[4] C. Blanchet-Scalliet And M. Jeanblanc: Hazard rate for credit risk and hedging defaultable contingent claims, Finance Stochast., 8 (2004) 145-159.

[5] P. Brémaud and M. Yor: Changes of filtration and of probability measures, Z.f.W, 45, (1978), 269-295.

[6] M. Bruche and C. Gonzlez-Aguado: Recovery rates, default probabilities, and the credit cycle, Journal of Banking \& Finance, 34(4), (2010), 754-764.

[7] U. Cetin, R. Jarrow, P. Protter and Y. Yildirim: Modeling Credit Risk with Partial Information. Annals of Applied Probability, 14(3), (2004,) 1167-1178.

[8] D. Coculescu, H. Geman and M. Jeanblanc: Valuation of default-sensitive claims under imperfect information, Finance Stochast., 12(2) (2008), 195-218.

[9] D. Coculescu, M. Jeanblanc and A. Nikeghbali: Default times, non arbitrage conditions and change of probability measures, Finance Stochast., 16(3) (2012), 513-535.

[10] D. Coculescu And A. Nikeghbali: Hazard processes and martingale hazard processes, Mathematical Finance, 22(3), (2012), 519-537.

[11] F. Delbaen And W. Schachermeyer: A general version of the fundamental theorem of asset pricing, Mathematische Annalen, 300(1), (1994) 463-520.

[12] C. Dellacherie: Capacités et processus stochastiques, Springer-Verlag (1972).

[13] C. Dellacherie And P.A. Meyer: Construction d'un processus prévisible ayant une valeur donnée dans un temps d'arrêt, Sém. Proba. XII (1978) 425-427.

[14] C. Dellacherie and P.A. Meyer: A propos du travail de Yor sur les grossissements des tribus, Sém.Proba. XII, Lecture Notes in Mathematics 649, (1978), 69-78.

[15] C. Dellacherie and P.A. Meyer: Probabilités et potentiel, Chapitres V-VIII: Théorie des Martingales, Hermann (1980).

[16] Duffie, D. And D. Lando: Term Structures of Credit Spreads with Incomplete Accounting Information, Econometrica, 69(3), (2001) 633-664.

[17] R.J. Elliott, M. Jeanblanc and M. Yor: On models of default risk, Math. Finance, 10, (2000) 179-196.

[18] R. Frey and T. Schmidt Pricing Corporate Securities under Noisy Asset Information, Math. Finance 19(3), (2009), $403-421$.

[19] F. Gehmlich and T. Schmidt: Dynamic Defaultable Term Structure Modelling Beyond the Intensity Paradigm, forthcoming Mathematical Finance.

[20] K. Giesecke and L. Goldberg: Forecasting Default in the Face of Uncertainty, Journal of Derivatives, 12(1), (2004) 14-25.

[21] X. Guo., R.A. Jarrow And Y. Zeng: Credit risk models with incomplete information, Mathematics of Operations Research, To appear.

[22] R. A. Jarrow and P. Protter (2004): Structural versus Reduced Form Models: A New Information Based Perspective, Journal of Investment Management, 2(2), 1-10.

[23] R. A. Jarrow P. Protter and D. Sezer (2007): Information Reduction via Level Crossing in a Credit Risk Model, Finance Stochast., 11(2), 195-212.

[24] M. Jeanblanc and M. Rutkowski: Modeling default risk: Mathematical tools, Fixed Income and Credit risk modeling and Management, New York University, Stern School of business, Statistics and Operations Research Department, Workshop (2000).

[25] T. Jeulin: Semi-martingales et grossissements d'une filtration, Lecture Notes in Mathematics 833, Springer (1980).

[26] T. Jeulin, M. Yor: Grossissement d'une filtration et semimartingales: formules explicites, Sém.Proba. XII, Lecture Notes in Mathematics 649, (1978), 78-97.

[27] T. Jeulin, M. Yor (EDS): Grossissements de filtrations: exemples et applications, Lecture Notes in Mathematics 1118, Springer (1985).

[28] Y. JiAO AND S. Li: Modelling sovereign risks: from a hybrid model to the generalized density approach to appear in Mathematical Finance.

[29] S. Kusuoka: A Remark on Default Risk Models, Advances on Mathematical Economics, 1, (1999) 69-82.

[30] Y. Le Jan: Temps d'arrêt stricts et martingales de saut, Z. Wahrscheinlichkeitstheorie verw. Gebiete, 44, (1978) $213-225$. Prob. 33, (2005) 1804-1824.

[31] A. Nikeghbali: An essay on the general theory of stochastic processes, Prob. Surveys, 3, (2006) 345-412.

[32] P.E. Protter: Stochastic integration and differential equations, Springer. Second edition (2005), version 2.1.

[33] M. Yor: Grossissements d'une filtration et semi-martingales: théorèmes généraux, Sém.Proba. XII, Lecture Notes in Mathematics 649, (1978), 61-69. 